Assessment of Fibrous Insulation Materials for the Selenide Isotope Generator System

\author{
G. C. Wei \\ V. J. Tennery
}




\section{DISCLAIMER}

This report was prepared as an account of work sponsored by an agency of the United States Government. Neither the United States Government nor any agency Thereof, nor any of their employees, makes any warranty, express or implied, or assumes any legal liability or responsibility for the accuracy, completeness, or usefulness of any information, apparatus, product, or process disclosed, or represents that its use would not infringe privately owned rights. Reference herein to any specific commercial product, process, or service by trade name, trademark, manufacturer, or otherwise does not necessarily constitute or imply its endorsement, recommendation, or favoring by the United States Government or any agency thereof. The views and opinions of authors expressed herein do not necessarily state or reflect those of the United States Government or any agency thereof. 


\section{DISCLAIMER}

Portions of this document may be illegible in electronic image products. Images are produced from the best available original document. 


\section{Printed in the United States of America. Available from National Technical Information Service \\ U.S. Department of Commerce 5285 Port Royal Road, Springfield, Virginia 22161 \\ Price: Printed Copy $\$ 4,50$; Microfiche $\$ 3.00$}

This report was prepared as an account of work sponsored by the United States Government. Neither the United States nor the Energy Research and Development Administration/United States Nuclear Regulatory Commission, nor any of their employees, nor any of their contractors, subcontractors, or their employees, makes any warranty, express or implied, or assumes any legal liability or responsibility for the accuracy, completeness or usefulness of any information, apparatus, product or process disclosed, or represents that its use would not infringe privately owned rights. 
Contract No. W-7405-eng-26

METALS AND CERAMICS DIVISION

\title{
ASSESSMENT OF FIBROUS INSULATION MATERIALS FOR THE SELENIDE ISOTOPE GENERATOR SYSTEM
}

\author{
G. C. Wei V. J. Tennery
}

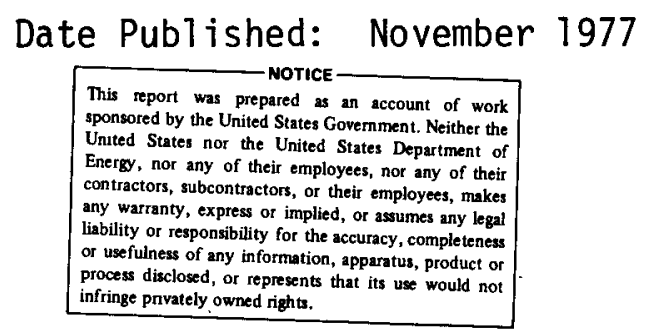

NOTICE This document contains information of a preliminary nature. It is subject to revision or correction and therefore does not represent a final report.

OAK RIDGE NATIONAL LABORATORY

Oak Ridge, Tennessee 37830

operated by

UNION CARBIDE CORPORATION

for the

DEPARTMENT OF ENERGY 
page blank 


\section{CONTENTS}

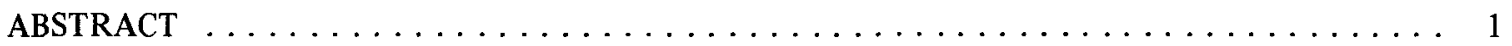

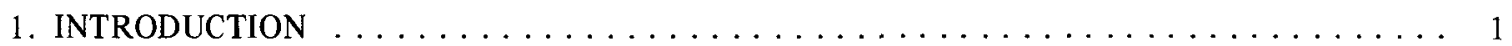

2. PRELIMINARY THERMAL INSULATION SYSTEM DESIGN AND MATERIAL SELECTION $\quad \ldots \quad 3$

3. CANDIDATE FIBROUS INSULATION MATERIALS $\ldots \ldots \ldots \ldots \ldots \ldots \ldots \ldots \ldots$

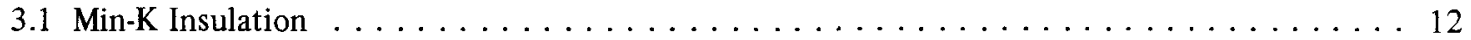

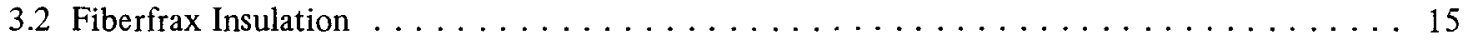

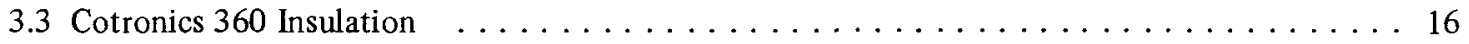

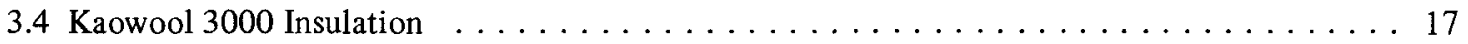

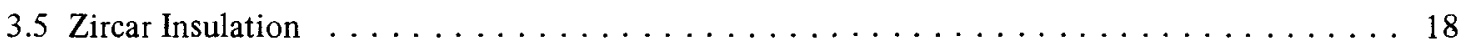

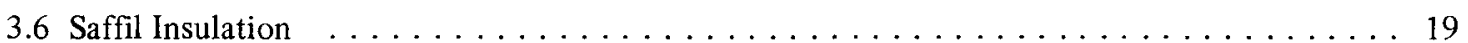

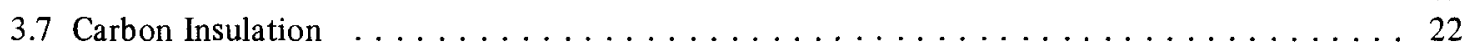

4. POTENTIAL APPLICATION PROBLEM AREAS $\ldots \ldots \ldots \ldots \ldots \ldots \ldots \ldots \ldots$

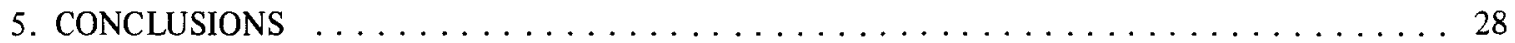




\title{
ASSESSMENT OF FIBROUS INSULATION MATERIALS FOR THE SELENIDE ISOTOPE GENERATOR SYSTEM
}

\author{
G. C. Wei V. J. Tennery
}

\begin{abstract}
This document reports an assessment of fibrous insulations for use in the converter and the heat source of the radiolsotope-powered, selenide element, thermoelectric generator (selenide ssotope generator) The most recent system design and material selection basis is presented. Several fibrous insulation materials which have the potential for use as load-bearing or nonload-bearing thermal insulations are reviewed, and thermophysical properties supplied by manufacturers or published in the literature are presented. Potential problems with the application of fibrous insulations in the selenide isotope generator are as follows compatibility with graplite, the thermoelectric elements, and the isolation hot frame; devitrification, grain growth, and sintering with an accompanying degradation of insulation quality; impurity diffusion from the insulation to adjoining structures, outgassing and storage of fibrous materials. Areas in which thermophysical data or quantitative information on the insulation and structural stability is lacking are identified.
\end{abstract}

\section{INTRODUCTION}

The selenide isotope generator is a radiosotope-powered thermoelectric converter energy system. The major components include an MHW (multi-hundred-watt) heat source and selenide thermoelectric elements. The heat generated by the isotopic heat source is converted to electricity through the selenide thermoelectric elements. A major milestone in the program, the final ground demonstration system (GDS) design review, is expected to be completed in June 1977. ${ }^{1}$ Following this ground demonstration, a second ground demonstration test may be performed under conditions simulating the proposed flight system. The first space missions using selenide isotope generators are scheduled for 1981. The characteristics of these systems are shown in Table 1.

In the preliminary design of the selenide isotope generator, the design heat loss through the thermal insulation is estimated as $10 \%{ }^{1}$ Anticipated achievement of the goal of a thermal efficiency of $90 \%$ is to be accomplished by use of highefficiency thermoelectric elements and through critical selection and design of

1. Low Cost High Performance Generator Technology Program Report, TES-3077-1, Teledyne Energy Systems (December 1976).

Table 1. Characteristics of the selenide isotope generator

\begin{tabular}{lcc}
\hline & \multicolumn{2}{c}{ Characteristics } \\
\cline { 2 - 3 } & Ground demonstration system & Proposed flight system \\
\hline Output power, W(e) & 100 & 261 \\
Load voltage, V(dc) & 10 & 29 \\
Specific power, W(e)/lb & 8.3 & 2.94 \\
Efficiency, \% & 1200 (electrically heated) & 10.5 \\
Fuel inventory (thermal power), W & & 2500 \\
Weight, lb & Helium & 88.84 \\
Heat source cover atmosphere & Xenon $(0.9$ atm) & Vacuum \\
Conver ter atmosphere & 850 & Vacuum \\
Hot-junction temperature, ${ }^{\circ} \mathrm{C}$ & 125 & 900 \\
Cold-junction temperature, ${ }^{\circ} \mathrm{C}$ & & 125 \\
\hline
\end{tabular}


the thermal insulation It is obvious that even small increases in the heat transport in the insulation material can lead to significant additional heat loss and, consequently, a significant reduction in output power The successful operation of the selenide isotope generator in terms of good economics and high performance as orignally proposed, therefore, depends critically on the effectiveness of the thermal insulation system, including the thermal insulation, insulation support structure, and gases within the insulation system.

In recent years, many high-temperature fibrous insulation materials have been developed ${ }^{2}$ The fibers are typically made of ceramic oxides such as $\mathrm{SiO}_{2}, \mathrm{Al}_{2} \mathrm{O}_{3}, \mathrm{Al}_{2} \mathrm{O}_{3}-\mathrm{SiO}_{2}, \mathrm{ZrO}_{2}$, or carbons These insulation materials have very low reported thermal conductıvity primarily due to their large percentage porosity and fibrous structure Several fibrous insulations have been considered for use in the selenide generator for load-bearing or nonload-bearing applications. In these cases the insulation materials are required to maintain low thermal conductance, structural stability, and dimensional integrity at high temperatures $\left(>850^{\circ} \mathrm{C}\right)$ throughout a service life of seven years $(60,000 \mathrm{hr})$. There are no such long-term data avalable in the literature. Previous generator systems used in Viking missions employed Min-K 1301 as the thermal insulation in both the thermoelectric modules and ends of heat source, but the temperature at the hot face of the insulation was only about $580^{\circ} \mathrm{C}$.

The apparent thermal conductivity of a fibrous insulation depends on many factors including the following ${ }^{3}$ gaseous atmosphere, temperature, temperature gradient, emissivity of the fiber, hot- and cold-side materials, thickness, density $\left(\mathrm{g} / \mathrm{cm}^{3}\right)$, fiber density $\left(\mathrm{No} / \mathrm{cm}^{3}\right)$, fiber diameter, fiber length, fiber orientation distribution, shot content, and fiber thermal conductivity Structural changes in the insulation such as cracking, devitrification, sintering, and grain growth, which can occur in the fibers at high temperatures during long-term operation and can cause shrinkage, are generally detrimental to the insulation stability. The net result of all these changes is usually a higher effective thermal conductance for the fibrous material

Despite the fact that fibrous materials are widely used in many insulation applications, thermal conductivity data for fibrous materials are incomplete, especially at high temperatures, and the conduction mechanisms are not clearly understood. ${ }^{2}$ The manufacturer-supplied data are often of limited value for an accurate thermal design analysis where a change in heat flux of a few percent can be very significant to the performance of the system. The thermal conductivity measurement for fibrous insulation material is difficult. ${ }^{2}$ Sampling to obtain a representative specimen for measurement is nontrivial in these materials because fibrous insulations are somewhat more difficult to fabricate consistently (variability $> \pm 15 \%$ ) and are inherently inhomogeneous as opposed to insulations such as multifoll or evacuated-opacified systems Also, the time to reach a steady-state temperature distribution in the thermal conductivity specimen and measurement system is very long for these low-conductivity materials and may require several days, thus makıng the measurements relatively expensive. The physical contact between the hot or cold surface of the apparatus and the fibrous conductivity sample is often poorly defined and difficult to reproduce. Many fibrous insulations undergo property changes during measurement, particularly at high temperatures In order that the measured thermal conductivity be applicable in a conceptual design, the measurement apparatus should employ conditions which as much as possible simulate the actual application The temperature gradient within the insulation and the emissivity of the hot and cold surfaces should be the same as that intended for the application For practical purposes, a system thermal conductance

2 R G Donnelly, V J Tennery, D L McElroy, T G Godfrey, and J O Kolb, Industrial Thermal Insulation, an Assessment, ORNL/TM-5283 (1976).

3 Thermal Conductivity, ed R P Tye, chap 6, "Heat Transmission in Low-Conductivity Materıals," Academic Press, New York 1969 
determination which can include such considerations is probably more meaningful than a thermal conductivity measurement on an insulation sample.

This report constitutes an assessment of the available insulation materials considered relevant to the selenide generator. Thermophysical properties such as chemical composition, density, dimensional stability, maximum usable temperature, thermal conductivity, and heat capacity of some candidate fibrous insulations are reviewed. Attention is also directed to raw-material processing employed in manufacturing fibrous insulations, outgassing and storage of fibrous materials, and installation procedures. Areas in which more accurate data may be required include physical or mechanical properties. Chemical reactions between the insulation materials and various components in the selenide generator have the potential to degrade the insulation capability of fibrous materials and deteriorate the mechanical properties of critical metallic parts. Effects of impurities, structural changes, and microstructural developments on the insulation stability may be significant for long-term service. Potential problem areas such as those outlined above are analyzed and discussed in the present report. It is hoped that this publication can serve as a useful reference and guideline for the thermal insulation system design and material selection for the GDS and flight system of the selenide ișotope generator program.

\section{PRELIMINARY THERMAL INSULATION SYSTEM DESIGN AND MATERIAL SELECTION}

The performance characteristics of the selenide isotope generator GDS and proposed flight system according to a status report of December $1976^{1}$ are listed in Table 1.

About $10 \%(14 \mathrm{lb})$ of the total weight $(142 \mathrm{lb})$ is allotted for thermal insulation materials. This includes both the insulation at the ends of the heat source and the insulation for the thermoelectric modules and nonmodule areas.

Fibrous thermal insulations were recommended for use in the selenide isotope generator by a system contractor. The relative merits of using fibrous insulations instead of multifoil insulation systems are manyfold. ${ }^{4}$ The thermal insulation design based on fibrous materials has higher specific power, higher efficiency, and higher probability of achieving the goal of more than $10 \%$ efficiency. In addition, less insulation support structure development is needed to ensure the ground demonstration success in 1977. The fill gas can be selected with relatively few restrictions, and thus problems associated with the sublimation of thermoelectric elements and outgassing of the insulation materials become less critical. The design temperature for fibrous insulation systems is also more flexible. Finally, the cost of fibrous insulation is substantially lower than other types having acceptably low conductance values. All these factors indicate that fibrous insulation is more suitable than other candidate insulations for the selenide isotope generator.

The latest generator design employs a ring arrangement of thermoelectric modules. POCO graphite, ${ }^{5}$ which surrounds the isolation hot frame, is in contact with a sprayed alumina coating on a molybdenum hot shoe in the thermoelectric module. In the thermoelectric module, approximately 13 layers of Fiberfrax ${ }^{6}$ Hi Fi 660 paper $\left(12 \mathrm{lb} / \mathrm{ft}^{3}\right.$ or $\left.0.19 \mathrm{~g} / \mathrm{cm}^{3}\right)$ with a typical layer thickness of $1 / 32$ in. will be pressed into the module and will result in a total thickness of about 0.3 to 0.33 in. after a compression of approximately 20 vol $\%$ of the unloaded material. Fiberfrax Hi Fi 660 paper insulation surrounds the thermoelectric $\mathbf{P}$ and $\mathrm{N}$ legs (copper silver selenide and gadolinium selenide respectively). A layer of Min- $\mathrm{K}^{7}$

4. Low Cost High Performance Generator Technology Program Report, TES-3075-43, Teledyne Energy Systems (1975).

5. Product of POCO Graphite, Inc., Decatur, Tex

6. Product of Carborundum Co., Niagara Falls, N.Y.

7. Product of Johns-Manville Co., Denver, Colo. 
TE $1800\left(20 \mathrm{lb} / \mathrm{ft}^{3}\right.$ or $\left.0.32 \mathrm{~g} / \mathrm{cm}^{3}\right)$ with a total thickness of $1 \frac{1}{2} \mathrm{in}$. is used in nonmodule areas. Min-K TE 1800 is in contact with POCO graphite. The present design temperatures for the hot and the cold sides of the thermoelectric module insulations are 900 and $150^{\circ} \mathrm{C}$ respectively. In the nonmodule region, the design hot- and cold-side temperatures are 930 and $125^{\circ} \mathrm{C}$. Figure $1(a)$ and $(b)$ schematically outlines the dimensions of the insulations for the converter.

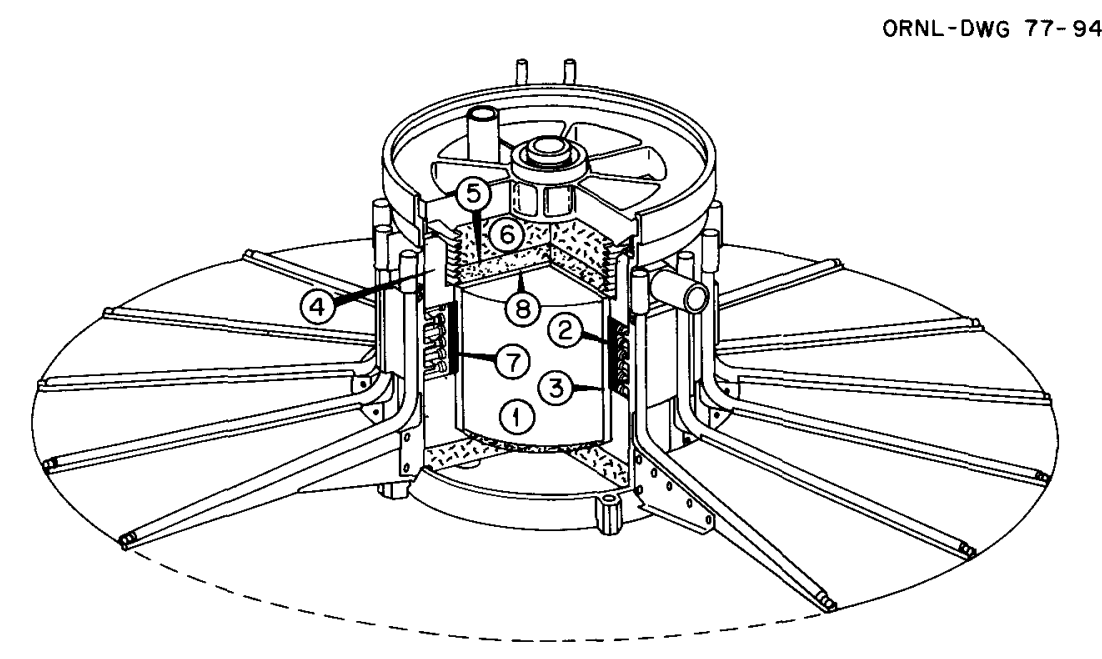

(1) ISOTOPIC HEAT SOURCE

(2) THERMOELECTRIC MODULE

(3) ISOLATION HOT FRAME

(a)

(4) SIDE INSULATION PADS
(5) END INSULATION - HIGH TEMPERATURE

(6) END INSULATION - LOW TEMPERATURE

(7) THERMOELECTRIC MODULE SUPPORT RING

(8) BORON NITRIDE ELECTRIC INSULATOR

(b)

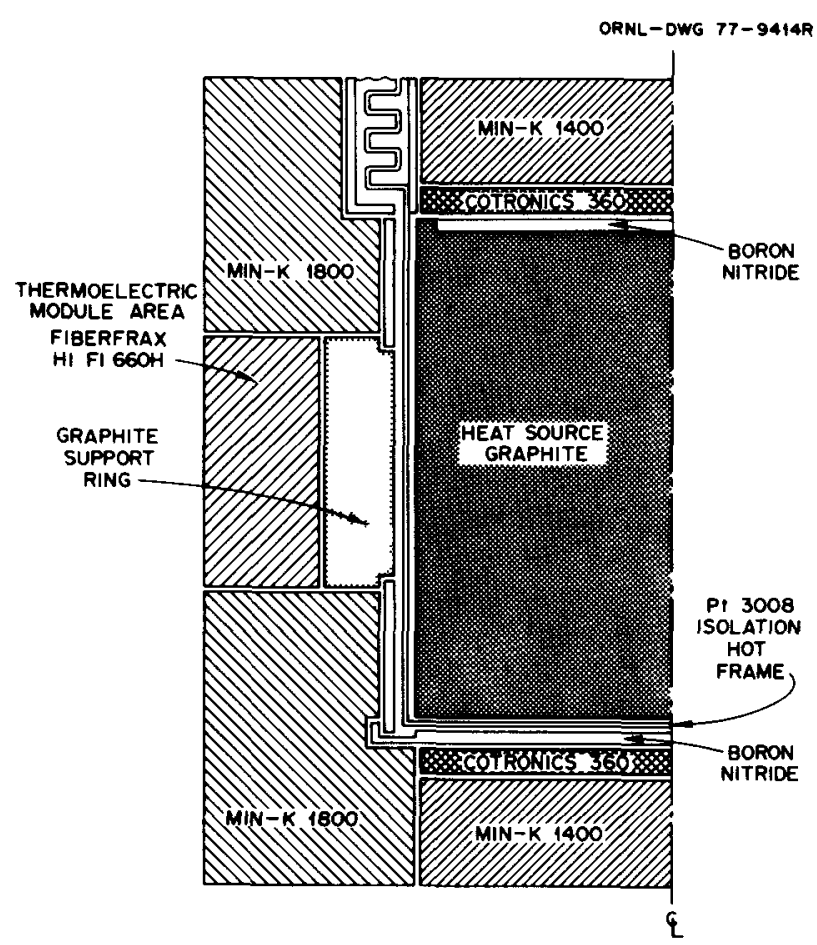

Fig. 1. Schematic outline of thermal insulation material design of the selenide isotope generator. 
At the ends of the heat source, the insulation thickness is $2 \mathrm{in} .(5.08 \mathrm{~cm})$. This is made of a layer of load-bearing insulation (presently unselected) supporting the hot end of the heat source and a layer of Min-K TE $1400\left(20 \mathrm{lb} / \mathrm{ft}^{3}\right.$ or $\left.0.32 \mathrm{~g} / \mathrm{cm}^{3}\right)$ at the cold side. The temperature at the ends of the heat source is greater than $930^{\circ} \mathrm{C}$. In the relatively hotter corner areas, Min-K 1800 is specified, but an additional high-temperature insulation layer (e.g., Zircar ${ }^{8}$ ) will be required. Figure 2 illustrates the configuration of the various insulations for the heat source.

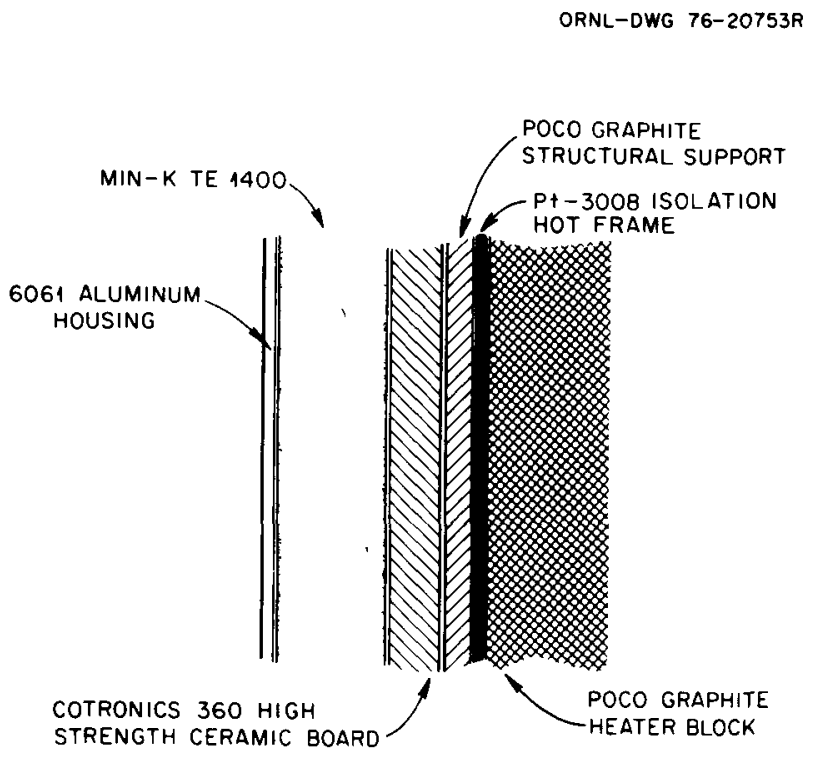

Fig. 2. Schematic outlines of heat source insulations.

In order to suppress the sublimation of the thermoelectric materials, xenon gas was considered for use in the converter. At the ends of the heat source, helium gas was selected among the candidate fill gases: helium, argon, and xenon. The presence of gas in fibrous insulation strongly influences the effective thermal conductivity. Because of the contribution of gas conduction and convection, the thermal conductivity of an inert gas-filled fibrous insulation can be orders of magnitude higher than that of an evacuated fibrous insulation.

The most suitable load-bearing insulation for use at the ends of the heat source and in the nonmodule areas of the converter has not been selected at this time. Insulation materials under consideration by the system contractor for this application are carbon foam, Kaowool $3000,{ }^{9}$ Cotronics $360,{ }^{10}$ and Zircar materials.

\section{CANDIDATE FIBROUS INSULATION MATERIALS}

In this section, several commercially available fibrous insulations potentially applicable to the selenide isotope generator will be discussed. Table 2 is a comprehensive list of various fibrous insulations with reported maximum usable temperatures above $500^{\circ} \mathrm{C}$, along with available composition and thermophysical

8. Product of Zircar Co., Florida, N.Y.

9. Product of Babcock and Wilcox, Augusta, Ga.

10. Product of Cotronics Co., New York, N.Y. 
Table 2. Fibrous insulation summary

\begin{tabular}{|c|c|c|c|c|c|c|c|c|}
\hline Insulation & Manutacturer & Composition & $\begin{array}{c}\text { Meltung } \\
\text { point } \\
\left({ }^{\circ} \mathrm{C}\right)\end{array}$ & $\begin{array}{l}\text { Maximum } \\
\text { rated } \\
\text { temperature } \\
\left({ }^{\circ} \mathrm{C}\right)\end{array}$ & $\begin{array}{l}\text { Density } \\
\left(\mathrm{lb} / \mathrm{ft}^{3}\right)\end{array}$ & $\begin{array}{c}\text { Fiber } \\
\text { dtameter } \\
(\mu \mathrm{m})\end{array}$ & $\begin{array}{c}\text { Fiber } \\
\text { length } \\
\text { (1n.) }\end{array}$ & Binder \\
\hline Kdowool bulk & Bdbcock and Wilcox & $\begin{array}{l}45 \% \mathrm{Al}_{2} \mathrm{O}_{3} \\
52 \% \mathrm{SlO}_{2}\end{array}$ & 1760 & 1260 & $3-12$ & 2.8 & 10 & None \\
\hline Kaowool blanket & & $\begin{array}{l}45 \% \mathrm{Al}_{2} \mathrm{O}_{3} \\
52 \% \mathrm{SlO}_{2}\end{array}$ & 1760 & 1260 & $3-8$ & 2.8 & 10 & None \\
\hline Kaowool block & & $\begin{array}{l}42 \% \mathrm{Al}_{2} \mathrm{O}_{3} \\
52 \% \mathrm{SlO}_{2}\end{array}$ & 1740 & 1200 & 12 & 2.8 & 0.5 & None \\
\hline Mullıte & & $\begin{array}{l}63 \% \mathrm{Al}_{2} \mathrm{O}_{3} \\
37 \% \mathrm{SIO}_{2}\end{array}$ & 1840 & 1540 & $3.2-7.7$ & 4 & 6 & \\
\hline XSW blanket & & $\begin{array}{l}50 \% \mathrm{Al}_{2} \mathrm{O}_{3} \\
47 \% \mathrm{~S}_{1} \mathrm{O}_{2}\end{array}$ & 1760 & 1260 & 6 & 2 & & None \\
\hline$X V$ felt & & $\begin{array}{l}50 \% \mathrm{Al}_{2} \mathrm{O}_{3} \\
47 \% \mathrm{SlO}_{2}\end{array}$ & 1760 & 1260 & 6 & 2 & & $1 \%$ \\
\hline Bulk tıber & & $\begin{array}{l}50 \% \mathrm{Al}_{2} \mathrm{O}_{3} \\
47 \% \mathrm{SiO}_{2}\end{array}$ & 1760 & 1260 & $3-4$ & 2 & to 1.5 & None \\
\hline Washed tiber & & $\begin{array}{l}50 \% \mathrm{Al}_{2} \mathrm{O}_{3} \\
47 \% \mathrm{~S}_{1} \mathrm{O}_{2}\end{array}$ & 1760 & 1260 & 4 & 2 & to 0.5 & None \\
\hline Chopped fiber & & $\begin{array}{l}50 \% \mathrm{Al}_{2} \mathrm{O}_{3} \\
47 \% \mathrm{SiO}_{2}\end{array}$ & 1760 & 1260 & 75 & 2 & 0.012 & None \\
\hline
\end{tabular}


Table 2. (continued)

\begin{tabular}{|c|c|c|c|c|c|c|c|c|}
\hline Insulation & Manufacturer & Composition & $\begin{array}{c}\text { Melting } \\
\text { point } \\
\left.{ }^{\circ} \mathrm{C}\right)\end{array}$ & $\begin{array}{l}\text { Maximum } \\
\text { rated } \\
\text { temperature } \\
\left({ }^{\circ} \mathrm{C}\right)\end{array}$ & $\begin{array}{l}\text { Density } \\
\left(\mathrm{l} b / \mathrm{ft}^{3}\right)\end{array}$ & $\begin{array}{c}\text { Fiber } \\
\text { diameter } \\
(\mu \mathrm{m})\end{array}$ & $\begin{array}{l}\text { Fiber } \\
\text { length } \\
\text { (in.) }\end{array}$ & Binder \\
\hline Lo-Con felt & & $\begin{array}{l}50 \% \mathrm{Al}_{2} \mathrm{O}_{3} \\
47 \% \mathrm{SiO}_{2}\end{array}$ & 1760 & 1260 & $4-6$ & 2 & 0.5 & $2 \%$ \\
\hline Block & & $\begin{array}{l}51 \% \mathrm{Al}_{2} \mathrm{O}_{3} \\
47 \% \mathrm{~S}_{1} \mathrm{O}_{2}\end{array}$ & 1760 & 1260 & 17 & 2 & NA & $2 \%$ \\
\hline Tubes & & $\begin{array}{l}51 \% \mathrm{Al}_{2} \mathrm{O}_{3} \\
47 \% \mathrm{SlO}_{2}\end{array}$ & 1760 & 1260 & 35 & 2 & 0.5 & $2 \%$ \\
\hline Lamınate & & $\begin{array}{l}51 \% \mathrm{Al}_{2} \mathrm{O}_{3} \\
47 \% \mathrm{SiO}_{2}\end{array}$ & 1760 & 1260 & 40 & 2 & 0.5 & $2 \%$ \\
\hline Vart-Form & & $\begin{array}{l}51 \% \mathrm{Al}_{2} \mathrm{O}_{3} \\
47 \% \mathrm{SiO}_{2}\end{array}$ & 1760 & 1310 & $30-80$ & & & $2 \%$ \\
\hline BN tiber & & $43 \% \mathrm{~B}, 56 \% \mathrm{~N}$ & 2650 & 2480 & $5-10$ & $5-7$ & $10-15$ & \\
\hline Paper & & $\begin{array}{l}50 \% \mathrm{Al}_{2} \mathrm{O}_{3} \\
47 \% \mathrm{SiO}_{2}\end{array}$ & 1760 & 1260 & 12 & 2 & 0.5 & $3 \%$ \\
\hline Fiberfrax $H_{1} F_{1} 660 \mathrm{H}$ & & $\begin{array}{l}51.7 \% \mathrm{Al}_{2} \mathrm{O}_{3} \\
47.6 \% \mathrm{~S}_{1} \mathrm{O}_{2}\end{array}$ & 1760 & 1260 & & 1.6 & to 0.5 & None \\
\hline Tipersul & E. I. du Pont & $\mathrm{K}_{2} \mathrm{Ti}_{6} \mathrm{O}_{13}$ & & 1200 & $3-24$ & 1 & 0.03 & \\
\hline Silica microspheres & Emerson and Cuming & $\mathrm{S}_{1} \mathrm{O}_{2}$ & & 1370 & $10-11$ & & & \\
\hline Glasrock & Glassrock & $99 \% \mathrm{SiO}_{2}$ & & 1100 & $25-106$ & & & \\
\hline Sil-Tem 25M & Harvey Industries & $98 \% \mathrm{StO}_{2}$ & 1650 & 1100 & 11 & 9 & NA & None \\
\hline Santocell A & $\begin{array}{l}\text { Monsanto Chemical } \\
\text { Corp. }\end{array}$ & $\mathrm{S}_{1} \mathrm{O}_{2}$ powder & & 700 & 5 & 0.025 & NA & \\
\hline Grayfoll & $\begin{array}{l}\text { High Temperature } \\
\text { Materials, Inc. }\end{array}$ & Carbon & & 3700 & 137 & & NA & \\
\hline Pyrotoam & & Carbon & & & $1-120$ & & $\mathrm{NA}$ & \\
\hline Mın-K 2000 & Johns-Manville & $\begin{array}{l}35 \% \mathrm{~S}_{1}, 48 \% \mathrm{O}_{2} \\
10 \% \mathrm{~T}_{1}\end{array}$ & & 1000 & 20 & & NA & Resin \\
\hline $\begin{array}{l}\text { High-density } \\
\text { Min-K } 2000\end{array}$ & & $\begin{array}{l}35 \% \mathrm{Si}, 48 \% \mathrm{O}_{2} \\
10 \% \mathrm{~T} 1\end{array}$ & & 1000 & $25,30,35$ & & NA & Resin \\
\hline
\end{tabular}


Table 2. (contınued)

\begin{tabular}{|c|c|c|c|c|c|c|c|c|}
\hline Insulation & Manufacturer & Composition & $\begin{array}{l}\text { Melting } \\
\text { point } \\
\left({ }^{\circ} \mathrm{C}\right)\end{array}$ & $\begin{array}{c}\text { Maximum } \\
\text { rated } \\
\text { temperdature } \\
\left({ }^{\circ} \mathrm{C}\right)\end{array}$ & $\begin{array}{l}\text { Density } \\
\left(\mathrm{lb} / \mathrm{ft}^{3}\right)\end{array}$ & $\begin{array}{c}\text { Fiber } \\
\text { diameter } \\
(\mu \mathrm{m})\end{array}$ & $\begin{array}{l}\text { Fiber } \\
\text { length } \\
\text { (1n ) }\end{array}$ & Binder \\
\hline $\begin{array}{l}\text { Flexıble } \\
\text { Min-K blankets }\end{array}$ & & & & 1000 & 16 & & NA & Resin \\
\hline Mın-K 1301 & & $52 \% \mathrm{~S}_{1}, 40 \% \mathrm{O}_{2}$ & & 700 & 20 & & NA & Resin \\
\hline Microquartz & & $99 \% \mathrm{SlO}_{2}$ & & 1200 & $3-6$ & 12 & NA & None \\
\hline Dynaquartz & & $99 \% \mathrm{SlO}_{2}$ & & 1500 & $45-10$ & 12 & NA & None \\
\hline Microquartz web & & $99 \% \mathrm{~S}_{10}$ & & 1200 & & & & \\
\hline Microquartz paper & & $99 \% \mathrm{SiO}_{2}$ & & & & & & \\
\hline Micro fibers felt $E$ & & Borosilicate & & 650 & 4 & 13 & & None \\
\hline Thermoflex felt & & $\begin{array}{l}42 \% \mathrm{Al}_{2} \mathrm{O}_{3} \\
50 \% \mathrm{SlO}_{2}\end{array}$ & 1760 & 1260 & $3-24$ & 3 & & $2 \%$ \\
\hline $\begin{array}{l}\text { Ceramic-bonded } \\
\text { Thermoflex }\end{array}$ & & $\begin{array}{l}21 \% \mathrm{Al}_{2} \mathrm{O}_{3} \\
77 \% \mathrm{SlO}_{2}\end{array}$ & 1760 & 1260 & $20-40$ & & & \\
\hline Microbestos pdper & & Asbestos fiber & & & & & & $3 \%$ organic \\
\hline Marınite & & Asbestos, $\mathrm{S}_{10}$ & & 650 & $23-36$ & & & Inorganic \\
\hline Dynaflex & & $\begin{array}{l}\mathrm{SlO}_{2}, \mathrm{Al}_{2} \mathrm{O}_{3} \\
\mathrm{Cr}_{2} \mathrm{O}_{3}\end{array}$ & 1650 & 1500 & $3-24$ & & & $3 \%$ \\
\hline Thermoflex web & & $\begin{array}{l}42 \% \mathrm{Al}_{2} \mathrm{O}_{3} \\
50 \% \mathrm{S1O}_{2}\end{array}$ & 1760 & 1260 & & & & None \\
\hline Marımet 45 & & $\mathrm{Ca}, \mathrm{S}_{1} \mathrm{O}_{2}$ & & 1000 & 44 & & & \\
\hline Zirconia foam & $\begin{array}{l}\text { National Beryllium } \\
\text { Corp }\end{array}$ & $\begin{array}{l}95 \% \mathrm{ZrO}_{2} \\
5 \% \mathrm{MgO}\end{array}$ & 2500 & 2300 & $50-87$ & & & \\
\hline Beryllıa fibers & & $\mathrm{BeO}$ & 2500 & 2100 & & $2-20$ & 0004 & \\
\hline Fiberglass & $\begin{array}{l}\text { Owen-Corning } \\
\text { Fiberglass }\end{array}$ & AA fibers & & 650 & $4-15$ & & & \\
\hline Fiberglass & & B fibers & & 650 & & & & \\
\hline Unıbestos & Pittsburgh Cornıng & Amosite asbestos & & 800 & 16 & & & \\
\hline Textrafluff & PPG & Glass fiber & & 540 & $\begin{array}{l}39,57, \\
76\end{array}$ & 10 & & None \\
\hline
\end{tabular}


Table 2. (continued)

\begin{tabular}{|c|c|c|c|c|c|c|c|c|}
\hline Insulation & Manufacturer & Composition & $\begin{array}{c}\text { Melting } \\
\text { point } \\
\left({ }^{\circ} \mathrm{C}\right)\end{array}$ & $\begin{array}{l}\text { Maximum } \\
\text { rated } \\
\text { temperature } \\
\left({ }^{\circ} \mathrm{C}\right)\end{array}$ & $\begin{array}{l}\text { Density } \\
\left(\mathrm{lb} / \mathrm{ft}^{3}\right)\end{array}$ & $\begin{array}{c}\text { Fiber } \\
\text { diameter } \\
(\mu \mathrm{m})\end{array}$ & $\begin{array}{c}\text { F1ber } \\
\text { length } \\
\text { (In) }\end{array}$ & Bınder \\
\hline Pyrold & Pyngenics, Inc & $\mathrm{C}$ & 3650 & 2900 & 137 & & & \\
\hline Microtherm & $\begin{array}{l}\text { Micropore Insulation } \\
\text { Ltd. }\end{array}$ & $\mathrm{S}_{2} \mathrm{O}_{2}$ & & 1000 & 20 & & & None \\
\hline WRP-X-AQ felt & Refractory Products Co. & $\begin{array}{l}51 \% \mathrm{Al}_{2} \mathrm{O}_{3} \\
47 \% \mathrm{SlO}_{2}\end{array}$ & 1760 & 1260 & 18 & & & \\
\hline Resistotherm & Resist Chemical Inc & $\mathrm{K}_{2} \mathrm{~T}_{6} \mathrm{O}_{13}$ & & 1200 & $3-28$ & & & \\
\hline Astroquartz & J. P. Stevens \& Co & $\mathrm{S}_{2}$ & 1650 & 1300 & $1-30$ & & & \\
\hline Astrosil & & $\mathrm{SiO}_{2}$ & 1650 & 980 & 3 & & & \\
\hline Sapphıre wool & $\begin{array}{l}\text { Thermo Kınetics } \\
\text { Fibers, Inc }\end{array}$ & $\mathrm{Al}_{2} \mathrm{O}_{3}$ & 2100 & 2040 & & $1-9$ & 025 & \\
\hline Refrasil Irish A1573 & H. I. Thompson & $96 \% \mathrm{SlO}_{2}$ & 1650 & 1300 & 35 & 1.3 & $1 / 8-1 / 4$ & Yes \\
\hline Refrasil Irish A1576 & & $96 \% \mathrm{SiO}_{2}$ & 1650 & 1300 & 35 & 10 & $1 / 4$ & Yes \\
\hline Refrasil batt A 100 & & $99 \% \mathrm{SiO}_{2}$ & 1730 & 1100 & 35 & 13 & $1 / 8-1 / 4$ & None \\
\hline Refrasll batt B100 & & $99 \% \mathrm{SiO}_{2}$ & 1730 & 1100 & & 10 & $1 / 4$ & None \\
\hline Refrasil cloth C-10028 & & $99 \% \mathrm{SiO}_{2}$ & 1730 & 1100 & & 10 & & None \\
\hline Refrasil cloth C-10048 & & $99 \% \mathrm{SiO}_{2}$ & 1730 & 1100 & & 13 & & None \\
\hline Refrasil cloth C-10096 & & $99 \% \mathrm{~S} 1 O_{2}$ & 1730 & 1100 & & 13 & & None \\
\hline Refrasll Fab-Bat B1570 & & $99 \% \mathrm{SiO}_{2}$ & 1730 & 1100 & & 13 & & None \\
\hline Refrasil fiber F-100A & & $99 \% \mathrm{SlO}_{2}$ & 1730 & 1100 & & 11 & $1 / 4-1 / 2$ & None \\
\hline Refrasil fiber F-100 & & $99 \% \mathrm{~S}_{1} \mathrm{O}_{2}$ & 1730 & 1100 & & 11 & 18 & None \\
\hline Zirconıa fiber A & & $93 \% \mathrm{ZrO}_{2}$ & 2500 & 1650 & & 10 & $1 / 8-1 / 2$ & $6 \% \mathrm{CaO}$ \\
\hline Zirconia fiber $E$ & & $85 \% \mathrm{ZrO}_{2}^{\prime}$ & 2500 & 1700 & & 10 & $1 / 8-1 / 2$ & $15 \% \mathrm{Nd}_{2} \mathrm{O}_{3}$ \\
\hline CCA-1 carbon cloth & & $95 \% \mathrm{C}$ & Sublımes & 3600 & & 10 & & None \\
\hline CFA $1 / 4$ carbon fibers & & $95 \% \mathrm{C}$ & Sublimes & 3600 & & 11 & 025 & None \\
\hline FT-25-7 carbon felt & & $95 \% \mathrm{C}$ & Sublimes & 3600 & & & 0.25 & None \\
\hline G-1550 graphite cloth & & $95 \% \mathrm{C}$ & Sublımes & 3600 & & 10 & -0.5 & None \\
\hline
\end{tabular}


Table 2. (continued)

\begin{tabular}{|c|c|c|c|c|c|c|c|c|}
\hline Insulation & Manufacturer & Composition & $\begin{array}{c}\text { Meltıng } \\
\text { point } \\
(C)\end{array}$ & $\begin{array}{l}\text { Maximum } \\
\text { rated } \\
\text { temperature } \\
\left({ }^{\circ} \mathrm{C}\right)\end{array}$ & $\begin{array}{l}\text { Density } \\
\left(\mathrm{lb} / \mathrm{ft}^{3}\right)\end{array}$ & $\begin{array}{c}\text { Fiber } \\
\text { diameter } \\
(\mu \mathrm{m})\end{array}$ & $\begin{array}{c}\text { Fiber } \\
\text { length } \\
(\text { In.) }\end{array}$ & Binder \\
\hline GHF felt & & E glass & 700 & 600 & $4-16$ & 13 & $1 / 8-1 / 6$ & None \\
\hline FA felt & & $\mathbf{F}$ glass & 700 & 600 & 45 & 13 & $1 / 8-1 / 4$ & None \\
\hline Zircar & Zircar & $\mathrm{ZrO}_{2}, \mathrm{HtO}_{2}, \mathrm{Y}_{2} \mathrm{O}_{3}$ & 2600 & 2500 & 20 & 6 & & None \\
\hline Saffil alumina & ICI, United States & $95 \% \mathrm{Al}_{2} \mathrm{O}_{3}, 5 \% \mathrm{~S}_{1} \mathrm{O}_{2}$ & 1982 & 1010 & 6 & 3 & \multicolumn{2}{|c|}{$7 \%$ rayon fibers } \\
\hline Saffil zirconia & & $92 \% \mathrm{ZrO}_{2}, 8 \% \mathrm{Y}_{2} \mathrm{O}_{3}$ & 2704 & 1093 & 6 & 3 & \multicolumn{2}{|c|}{$7 \%$ rayon fibers } \\
\hline Cotronics & Contronics Co. & $\mathrm{Al}_{2} \mathrm{O}_{3}-\mathrm{S}_{1} \mathrm{O}_{2}$ & 1760 & 1260 & & & \multicolumn{2}{|c|}{ Organic and inorganic } \\
\hline
\end{tabular}


property data, as well as their manufacturers. Figure 3 shows the thermal conductivity data in air of many fibrous insulation materials. These data were reported by the manufacturers. The thermal conductivities of several fibrous insulations in vacuum and in xenon and helium atmospheres $(0.9 \mathrm{~atm})$ measured by Dynatech R/D Co. are presented in Figs. 4, 5, and 6. The following commercial or semicommercial insulation materials are discussed in more detail in this section: Min-K, Fiberfrax, Cotronics 360, Kaowool 3000, Zircar, Saffil, ${ }^{11}$ and carbon insulations.

11. Product of U.S. ICI Co., Wilmington, Del.

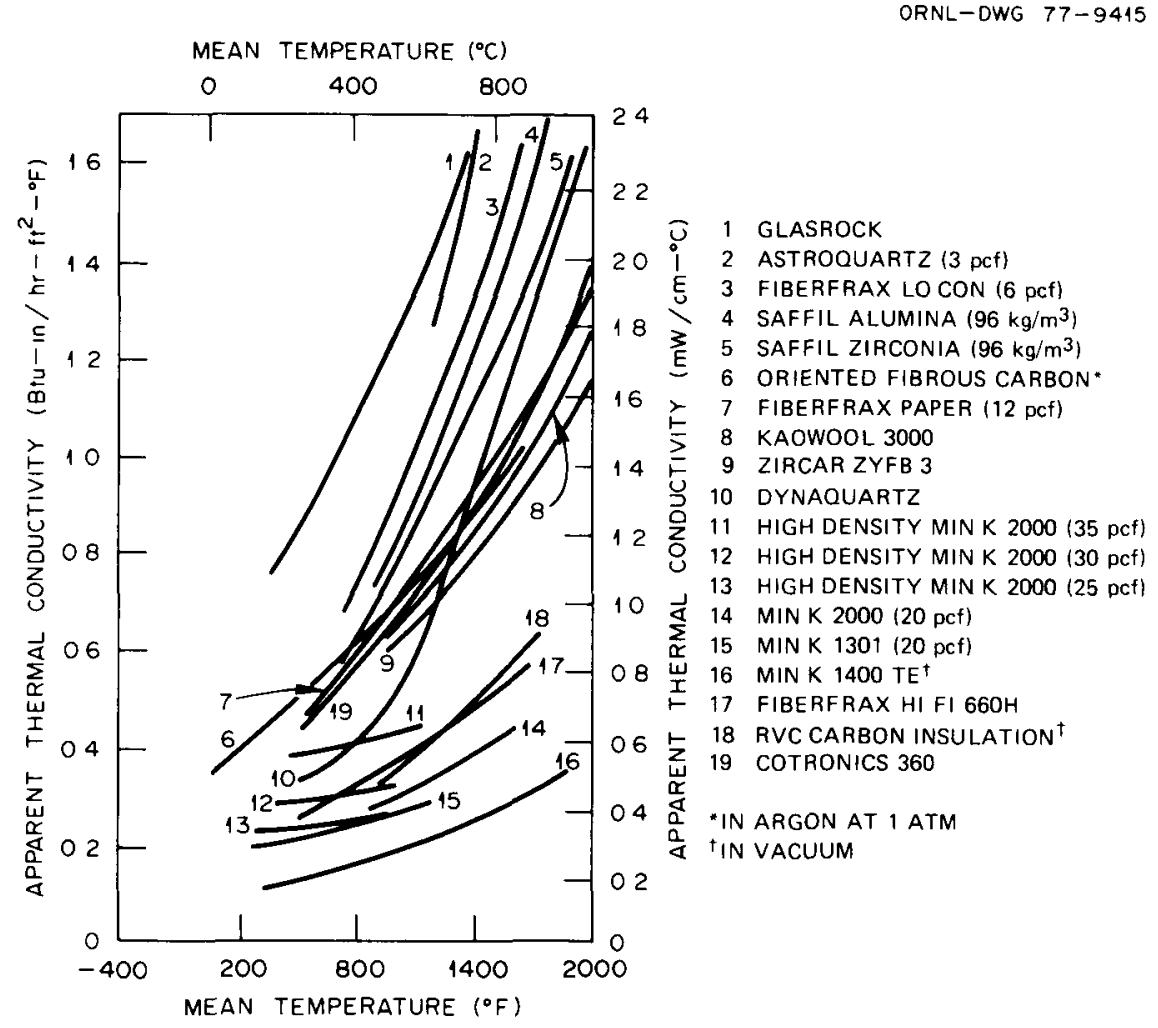

Fig. 3. Apparent thermal conductivity vs mean temperature for some fibrous insulation materials in air at 1 atm.

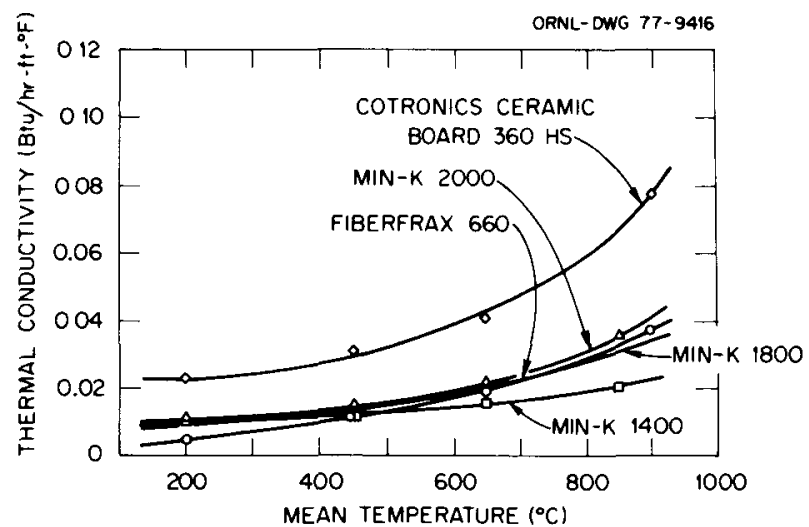

Fig. 4. Thermal conductivity of several fibrous insulations vs mean temperature in vacuum. Measured by Dynatech R/D Co. 


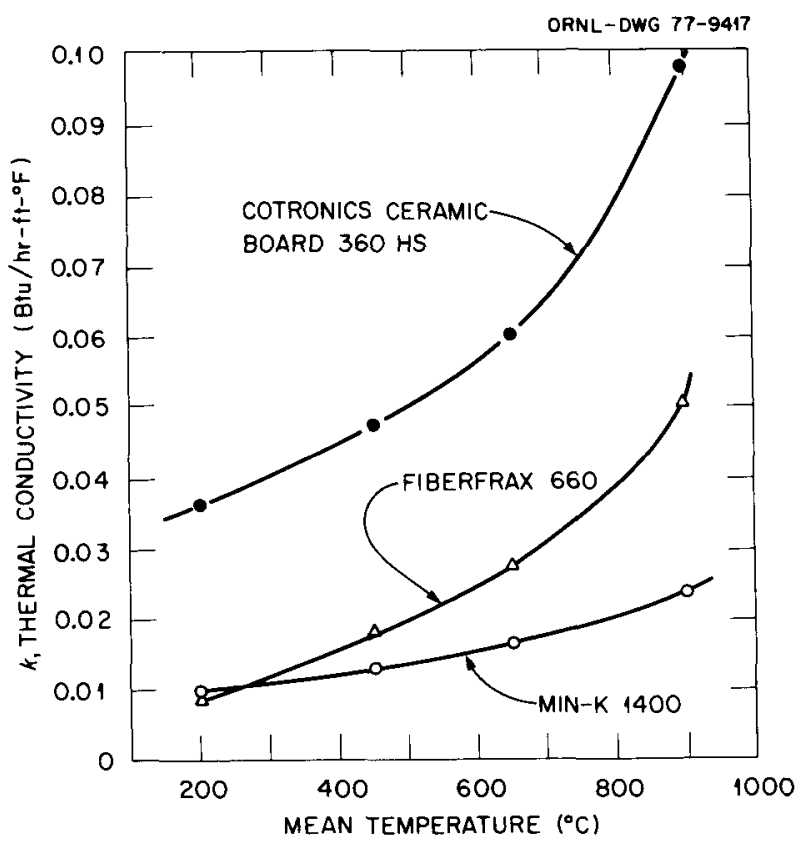

Fig. 5. Thermal conductivity of several fibrous insulations vs mean temperature in xenon. Measured by Dynatech R/D Co.

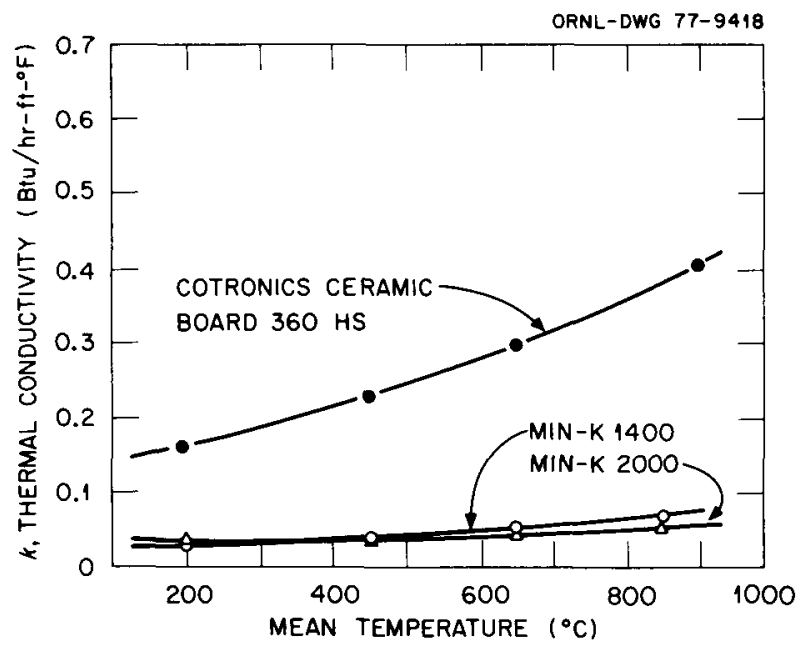
Co.

Fig. 6. Thermal conductivity of several fibrous insulations vs mean temperature in helium. Measured by Dynatech R/D

\subsection{Min-K Insulation}

The term Min-K represents a family of insulation materials developed and manufactured by the Johns-Manville Co. This family is widely known and has been used in many high-performance insulation applications such as manned spacecraft, radioisotope thermoelectric generators, and nuclear reactors. The application of Min- $\mathrm{K}$ in the radioisotope thermoelectric generator involved a maximum temperature, $580^{\circ} \mathrm{C}$, at the hot face of the insulations. Min- $\mathrm{K}$ insulation consists of submicron fused silica particles 
combined with a minor concentration of an opacifier $\left(\mathrm{T}_{1} \mathrm{O}_{2}\right)$ plus glass fibers as structural reinforcement Early Min-K products contain asbestos fibers and some binders The low thermal conductivity of Min-K materials is primarily due to the numerous extremely fine pores (in the range of the mean free path of air) associated with the fine silica particles The size and distribution of porosity in Min-K materials may need optımization for applications involving gaseous atmospheres other than air or vacuum The silica raw material is Cab-O-Sil M5, ${ }^{12}$ which has a particle size of about 15 to $20 \mathrm{~m} \mu \mathrm{m}$ The chemical compositions of Cab-O-Sil M5 and two other silica raw materials, Santocel $\mathrm{A}^{13}$ and Arc Silica 800, ${ }^{14}$ are listed in Table 3 A

Table 3. Spectrographic analysis of Santocel A,

Cab-O-Sil M5, and Arc Silica 800 used

in manufacturing Mın-K materials

\begin{tabular}{|c|c|c|c|}
\hline \multirow{2}{*}{ Metal } & \multicolumn{3}{|c|}{ Andilysis $(\%)$} \\
\hline & Santocel A & $C_{d} b-O-S 1 l$ M5 & Arc Siluca 800 \\
\hline Aluminum & 0048 & $<001$ & 0025 \\
\hline Boron & $<001$ & $<001$ & $<001$ \\
\hline Calcrum & $<001$ & $<001$ & $<001$ \\
\hline Iron & 0014 & $<001$ & $<001$ \\
\hline Magnesium & $<001$ & $<001$ & $<001$ \\
\hline Sodium ${ }^{a}$ & 031 & $<005$ & $<005$ \\
\hline Titanium & 0020 & $<001$ & $<001$ \\
\hline Zirconium & $<001$ & $<001$ & $<001$ \\
\hline
\end{tabular}

$a_{0} 05 \%$ represents the minımum estımate pussible by this andlysis

thermogravimetric analysis of Cab-O-Sil M5 indicated that a product made from it might be expected to give off only 025 to $0.55 \%$ moisture at temperatures up to $705^{\circ} \mathrm{C}\left(1300^{\circ} \mathrm{F}\right)$, if properly dried prior to testing ${ }^{15}$ Pure silica fibers such as Astroquartz ${ }^{16}$ and Microquart $L^{7}$ are used as fiber reinforcement in this insulation. Irish Refrasil ${ }^{17}$ is a chromum-treated silica fibrous material and is not recommended for use in the selenide generator because of possible contamination of generator parts with chromium. The $\mathrm{TiO}_{2}$ opacifier used in the Min-K products is $-5-\mu$ H.G. $\mathrm{T}_{1} \mathrm{O}_{2},{ }^{18}$ the spectrographic analysis of which is presented in Table 4 The major impurities in the opacifier are silicon, antimony, and aluminum

Detals of the procedures for manufacture of Min-K materials are considered propnetary information Procedures published in the open literature for these materials are outlined in the following text Starting materials for fabricating Min-K are mixed in a ribbon mixer and then passed through a comminuting machine for fiber dispersion. Steel dies are used for molding Min-K to the desired shape No binders are used, but an adequate coatıng of a mold release agent is used. The molding is done at room temperature,

12 Product of G L Cabot Co, Kokomo, Ind

13 Product of Monsanto Co, St Louis, Mo

14 Product of Pittsburgh Plate Glass Co, Pittsburgh, Pa

$15 \mathrm{~J}$ O Colluns, Develop I800F 400F Fibrous Type Insulation for Radiotsotope Power Systems, phase 1 final report, vol 1, ALO-3633-7 (1967)

16 Product of J P Stevens and Co, New York, N Y

17 Product of H I Thompson Co, Gardena, Calif

18 Product of TAM Division of National Lead Co, New York, N Y 


\begin{tabular}{lc}
$\begin{array}{c}\text { Table 4. Spectrographic analysis of } \\
\text { H.G. TiO } \\
\text { manufacturing Min-K materials }\end{array}$ \\
\hline \multicolumn{1}{c}{ Metal } & Content (\%) \\
\hline Alumınum & 0.14 \\
Antımony & 0.21 \\
Barıum & 0.014 \\
Iron & 0.018 \\
Ledd & 0.01 \\
Magnesıum & 0.01 \\
Silıcon & 0.23 \\
Zirconıum & 0.03 \\
\hline
\end{tabular}

and pressure is maintained for about $1 \mathrm{hr}$. Subsequent firing of the molded product then produces the final Min-K insulation.

Min- $\mathrm{K}$ materials are available in many different grades (different maximum usable temperature) and forms (sheet, tape, and flexible batts). A summary of thermophysical properties of Min-K 503, Min-K 1200, Min-K 1301, Min-K 1400 TE, Min-K 1800, and Min-K 2000 is listed in Table 5. The linear dimensional changes of Min- $\mathrm{K} 2000\left(22.2 \mathrm{lb} / \mathrm{ft}^{3}\right.$ or $\left.0.355 \mathrm{~g} / \mathrm{cm}^{3}\right)$ have been measured and are -5 and $-35 \%$ at 982 and $1093^{\circ} \mathrm{C}$ for a $24-\mathrm{hr}$ exposure. ${ }^{15}$ Thermal conductivity data in vacuum and in helium for Min-K $1400 \mathrm{TE}$ supplied by the manufacturer and measured recently by a contract measurement laboratory ${ }^{19}$ are

19. Dynatech R/D Co., Cambridge, Mass., under contracts from Teledyne Energy Systems, Tımonı, Md. (supported by ERDA).

Table 5. Typical properties of Min-K products

\begin{tabular}{|c|c|c|c|c|c|c|}
\hline Physical property & Mın-K 503 & Mın-K 1200 & Min-K 1301 & Min-K $1400 \mathrm{TE}$ & Min-K 1800 & Min-K 2000 \\
\hline $\begin{array}{l}\text { Maximum service temperature, } \\
{ }^{\circ} \mathrm{F}\end{array}$ & 500 & 1200 & 1300 & 1400 & 1800 & 1800 \\
\hline Nominal density, $\mathrm{lb} / \mathrm{ft}^{3}$ & 15 & 20 & 20 & 20 & 20 & 20 \\
\hline \multicolumn{7}{|l|}{ Compressive strength, psi } \\
\hline $5 \%$ compression & 45.0 & Nonload bearıng & 950 & 75 & Nonload bearıng & 80 \\
\hline $10 \%$ compression & 100 & & 200.0 & 145 & & 170 \\
\hline \multicolumn{7}{|l|}{$\begin{array}{l}\text { Thermal conductivity (in arr), } \\
\text { Btu } \times 1 \mathrm{n} . / \mathrm{ft}^{2} \times \mathrm{hr} \times{ }^{\circ} \mathrm{F}\end{array}$} \\
\hline $200^{\circ} \mathrm{F}(\operatorname{mean})$ & 0.18 & & & & & \\
\hline $300^{\circ} \mathrm{F}$ & 019 & & 021 & & & \\
\hline $400^{\circ} \mathrm{F}$ & 020 & & 0.22 & & & \\
\hline $600^{\circ} \mathrm{F}$ & & 0.21 & 0.23 & 0.21 & 0.21 & \\
\hline $800^{\circ} \mathrm{F}$ & & 0.23 & 0.25 & 0.23 & 023 & 0.27 \\
\hline $1000^{\circ} \mathrm{F}$ & & 0.25 & 027 & 025 & 0.25 & 030 \\
\hline $1200^{\circ} \mathrm{F}$ & & & & 0.27 & & 034 \\
\hline $1400^{\circ} \mathrm{F}$ & & & & & & 039 \\
\hline $1600^{\circ} \mathrm{F}$ & & & & & & 046 \\
\hline \multicolumn{7}{|l|}{ Specifıc heat, Btu/lb $\cdot{ }^{\circ} \mathrm{F}$} \\
\hline $400^{\circ} \mathrm{F}$ & & & 023 & & & 023 \\
\hline $800^{\circ} \mathrm{F}$ & & & 0.25 & & & 0.25 \\
\hline $1200^{\circ} \mathrm{F}$ & & & 0.27 & & & 0.27 \\
\hline $1600^{\circ} \mathrm{F}$ & & & 0.27 & & & 027 \\
\hline
\end{tabular}


compared in Fig. 7(a) and (b). At high temperatures, the measured data and the manufacturer's data are different by a factor of 2, but within the range of its usable temperature, Min-K products have one of the lowest known thermal conductivities.

(a)
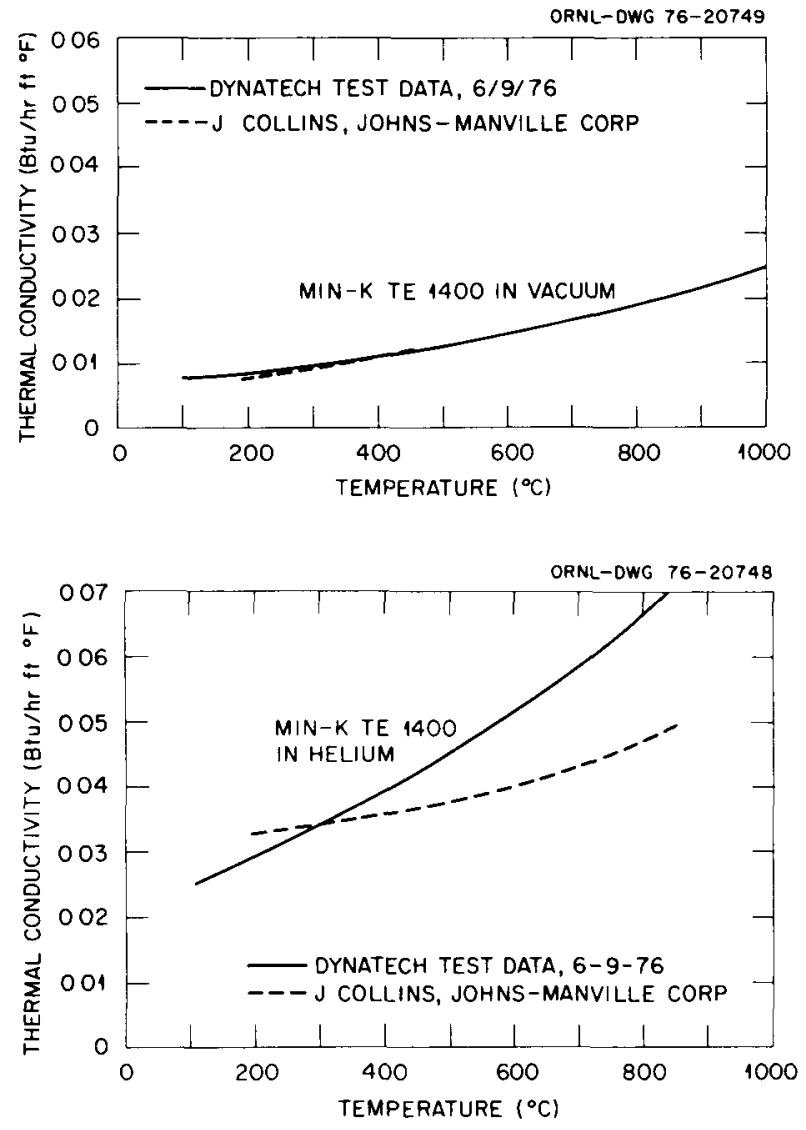

Fig. 7. Thermal conductivity of Min-K TE 1400 as a function of mean temperature $(a)$ in vacuum and $(b)$ in helium.

\subsection{Fiberfrax Insulation}

Fiberfrax is a family of alumino-silicate fibrous insulations produced by the Carborundum Company They are basically amorphous alumina-silica fibrous materials. The particular type of Fiberfrax product which will be discussed in this section is Fiberfrax $\mathrm{H}_{1} \mathrm{~F}_{1} 660$ paper. This fibrous insulation is made from Fiberfrax washed fibers in which the unfiberized shot has been removed. The average fiber diameter is 1.6 $\mu \mathrm{m}$, and the average fiber length is $05 \mathrm{in}(1.27 \mathrm{~cm})$. The final product is produced with or without organic binders. According to the manufacturer, series $660 \mathrm{H}$ paper is completely inorganic. Recently, it has been discovered that, in contrast to the manufacturer's data, Fiberfrax $\mathrm{H}_{1} \mathrm{Fl}_{1} 660 \mathrm{H}$ does contain organic binders. ${ }^{20}$ Complete removal of these organic binders requires heat treatment at high temperatures in air and subsequent outgassing at high temperatures as well. A typical chemical analysis is shown in Table 6 . The major cation impurities reported by the manufacturer are $\mathrm{Na}, \mathrm{B}$, and $\mathrm{Fe}$ Figure 8 illustrates the

20 Private communication trom R Ericson, 3M Co, St Paul, Minn 
Table 6. Typical chemical analysis of

Fiberfrax $\mathrm{H}_{1} \mathrm{~F}_{1} 660 \mathrm{H}$ paper and

Fiberfrax $\mathrm{H}$ blanket

\begin{tabular}{lcc}
\hline \multirow{2}{*}{ Content equivalent } & \multicolumn{2}{c}{ Analysis (wt \%) } \\
\cline { 2 - 3 } & $\mathrm{H}_{1} \mathrm{~F}_{1} 660 \mathrm{H}$ & H blanket \\
\hline $\mathrm{Al}_{2} \mathrm{O}_{3}$ & 517 & 622 \\
$\mathrm{SiO}_{2}$ & 476 & 363 \\
$\mathrm{Na}_{2} \mathrm{O}$ & 03 & $a$ \\
$\mathrm{~B}_{2} \mathrm{O}_{3}$ & 015 & 08 \\
$\mathrm{Fe}_{2} \mathrm{O}_{3}$ & 002 & $a$ \\
$\mathrm{Trace}_{\text {inorganics }}$ & 02 & $a$ \\
Ledchable chlorides & $<50 \mathrm{ppm}$ & $a$ \\
\hline
\end{tabular}

${ }^{a}$ Content unknown

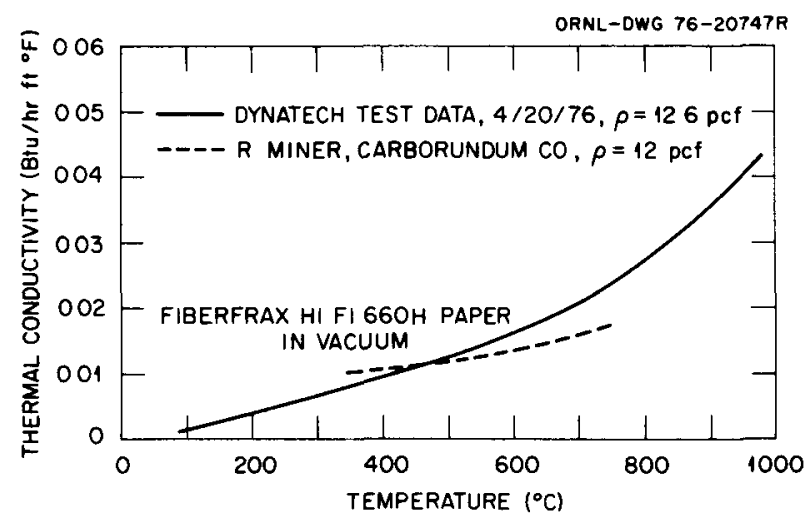

Fig. 8. Thermal conductivity of Fiberfrax $660 \mathrm{AH}$ paper in vacuum as a function of mean temperature.

thermal conductivity of Fiberfrax $\mathrm{H}_{1} \mathrm{~F}_{1} 660 \mathrm{H}$ measured in vacuum by the manufacturer and by Dynatech R/D Co., and Table 7 lists typical physical properties. Strauss ${ }^{21}$ evaluated the weight and dimensional changes in Fiberfrax insulations after exposure to various temperatures in a gas-fired furnace. His results are summarized in Fig. 9.

Recently, the production of $\mathrm{H}_{1} \mathrm{~F}_{1} 660$ paper was halted, and the manufacturer recommended substitution of Fiberfrax 970 series paper, which has a $37 \%$ higher thermal conductivity than does the Fiberfrax $\mathrm{Hi} \mathrm{F}_{1} 660 \mathrm{H}$ product. Fiberfrax $\mathrm{H}$ blanket is also avalable as an alternate insulation material to replace Fiberfrax $\mathrm{Hi}_{1} \mathrm{~F}_{1} 60 \mathrm{H}$. The chemical analysis and physical properties of Fiberfrax $\mathrm{H}$ blanket are also shown in Tables 6 and 7 respectively It should be noted that Fiberfrax $\mathrm{H}$ blanket has 0.8 wt $\% \mathrm{~B}_{2} \mathrm{O}_{3}$ equivalent content.

\subsection{Cotronics 360 Insulation}

Cotronics is a trade name of a family of alumino-silicate fibrous insulation materials made by Cotronics Corporation, New York, N.Y. Cotronics 360 ceramic board is a rigid, fibrous material selected in the

21 E L. Strauss, Evaluation of Low Cost/High Temperature Insulation, NASA-CR-134902 (1975) 
Table 7. Typical properties of Fiberfrax Hi Fi paper and Fiberfrax $\mathrm{H}$ blanket

\begin{tabular}{ccc}
\hline \multirow{2}{*}{ Physicdl property } & \multicolumn{2}{c}{ Characteristics } \\
\cline { 2 - 3 } & Hi F1 660 & H blanket \\
\hline Average fiber didmeter, $\mu \mathrm{m}$ & 1.6 & 24 \\
Average fiber length, cm & $<127$ & $<1.27$ \\
Mdximum usdble temperature, ${ }^{\circ} \mathrm{F}$ & 2300 & 2600 \\
Melting temperature, ${ }^{\circ} \mathrm{F}$ & 3200 & $>3500$ \\
Thermal conductivity (in arr), & & \\
Btu $\times$ in. $/ \mathrm{ft}^{2} \times \mathrm{hr} \times{ }^{\circ} \mathrm{F}$ & & \\
$600^{\circ} \mathrm{F}$ (mean) & 0.25 & 0.48 \\
$800^{\circ} \mathrm{F}$ & 0.30 & 0.63 \\
$1000^{\circ} \mathrm{F}$ & 0.35 & 0.79 \\
$1200^{\circ} \mathrm{F}$ & 0.41 & 0.96 \\
$1400^{\circ} \mathrm{F}$ & 0.51 & 1.16 \\
$1600^{\circ} \mathrm{F}$ & 0.61 & 138 \\
$1800^{\circ} \mathrm{F}$ & 0.74 & 1.60 \\
& &
\end{tabular}

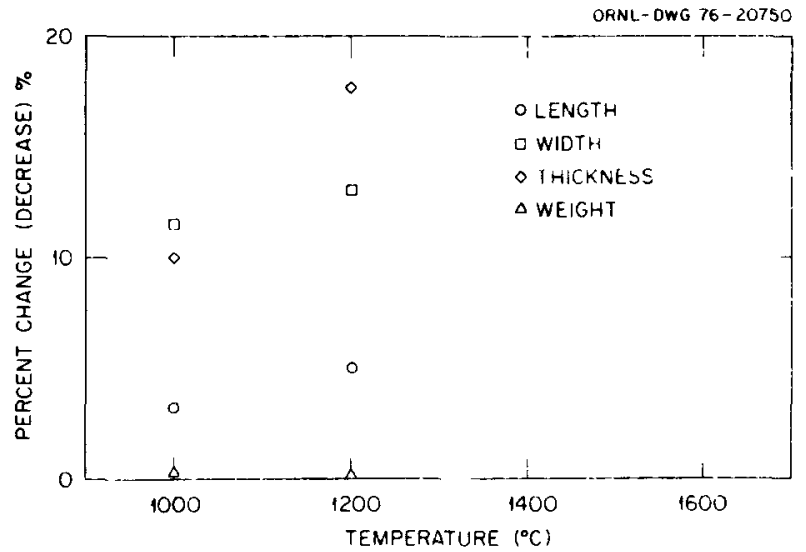

Fig. 9. Weight and dimensional changes in Fiberfrax $H$ blanket after 100-hr exposure to various ternust $1 \mathrm{r} . ., 4$

preliminary design for thermal insulation application in the selende isotope generator fin inorgatil $h_{1} \cdot$. was used as the bonding material for the alumino-silicate fibers in Cotronics 360 cerame board. In adduru: it contains substantial amounts of impurities. The potential problems include long-term stabiliz;, $\mathrm{d}$ compatibility with other material in the selenide isotope generator; it is questionable whether Cutr. $n_{1}$ s 360 is suitable for this thermal insulation application. The bulk density is $16 \mathrm{lb} / \mathrm{ft}^{3}\left(0.26 \mathrm{~g} / \mathrm{cm}^{3}\right)$ Compressive strength is reported by the manufacturer as 350 psi. Typical properties of the Cotronics $30 y$ ceramic board are listed in Table 8.

\subsection{Kaowool 3000 Insulation}

Kaowool is a trade name of a family of fibrous materials made by Babcock and Wilcux Company 6

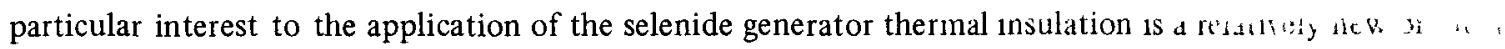
designated Kaowool 3000 board. This ceramic board is based on Saffl fjbes $=\langle 7, ;$ : , , bonded with some organic plus inorganic binders. The density is in the range $c t 1,1, \ldots+1$. 
Table 8. Typical properties of Cotronics 360 ceramic board

\begin{tabular}{ll}
\hline \multicolumn{1}{c}{ Physical property } & Characteristics \\
\hline Melting point, ${ }^{\circ} \mathrm{F}$ & 3200 \\
Contınuous use temperature, ${ }^{\circ} \mathrm{F}$ & 2300 \\
Density $\mathrm{lb} / \mathrm{ft}^{3}$ & 16 \\
Specif $\mathrm{F}$ hedt, Btu $/ \mathrm{lb} \cdot{ }^{\circ} \mathrm{H}$ & 025 \\
Modulus of rupture psi & 55 \\
Compressive strength psi & 350 \\
Mean thermal conductivity (in aur), & \\
Btu $\times$ in $/ \mathrm{ft}^{2} \times \mathrm{hr} \times{ }^{\circ} \mathrm{F}$ & \\
$500^{\circ} \mathrm{F}$ & 045 \\
$1000^{\circ} \mathrm{F}$ & 070 \\
$1500^{\circ} \mathrm{F}$ & 095 \\
$2000^{\circ} \mathrm{F}$ & 155 \\
\hline
\end{tabular}

$\left.019 \mathrm{~g} / \mathrm{cm}^{3}\right)$ The compressive strength is cited as $5 \%$ deformation at $1500 \mathrm{psf}\left(718 \times 10^{4} \mathrm{~Pa}\right)$ Linear shrinkage is given by the manufacturer as $15 \%$ at $1538^{\circ} \mathrm{C}\left(2800^{\circ} \mathrm{F}\right)$ The relevant physical properties of this material are listed in Table 9 The thermal conductivity vs mean temperature reported by the manufacturer is shown in Fig 10 Its applicability to the thermal insulation system of the selenide generator needs experimental justification of its reported thermal conductivity and mechanical strength

\section{Zircar Insulation}

Zircar is a trade name of a series of fibrous insulation products made by Zircar Products, Inc Basically, they are stabilized zırconıa fibrous materials Among varıous Zircar materials currently avarlable, zırconia board type ZYFB3 is a rigid, fibrous material probably suitable for thermal insulation application in the selenide generator It is zirconia stabilized with $12 \mathrm{wt} \%$ yttria, it contains no organic binders The density is $30 \mathrm{lb} / \mathrm{ft}^{3}\left(048 \mathrm{~g} / \mathrm{cm}^{3}\right)$ or $60 \mathrm{lb} / \mathrm{ft}^{3}\left(096 \mathrm{~g} / \mathrm{cm}^{3}\right)$ The linear shrinkage is reported by the manufacturer as $1 \%$ at $300^{\circ} \mathrm{F}$ The physical properties of the Zircar board (ZYFB3) and the fibers used in this board reported by the manufacturer are listed in Table 10 The thermal conductivity vs mean temperature in air cited in the manufacturer's catalog is shown in Fig 11

Table 9. Typical properties of Kaowool 3000

\begin{tabular}{|c|c|}
\hline Physical property & Characteristics \\
\hline Color & White \\
\hline Density, $\mathrm{lb} / \mathrm{ft}^{3}$ & $10 \quad 12$ \\
\hline Melting point, ${ }^{\circ} \mathrm{F}$ & 3400 \\
\hline Modulus of rupture, psi & 65 \\
\hline $\begin{array}{l}\text { Compressive strength, psf } \\
5 \% \text { deformation } \\
10 \% \text { deformation }\end{array}$ & $\begin{array}{l}1500 \\
2500\end{array}$ \\
\hline $\begin{array}{l}\text { Linear shrunkage } \%(\operatorname{mdx})^{a} \\
\qquad \begin{array}{l}2800^{\circ} \mathrm{F} \\
3000^{\circ} \mathrm{F}\end{array}\end{array}$ & $\begin{array}{l}15 \\
30\end{array}$ \\
\hline
\end{tabular}

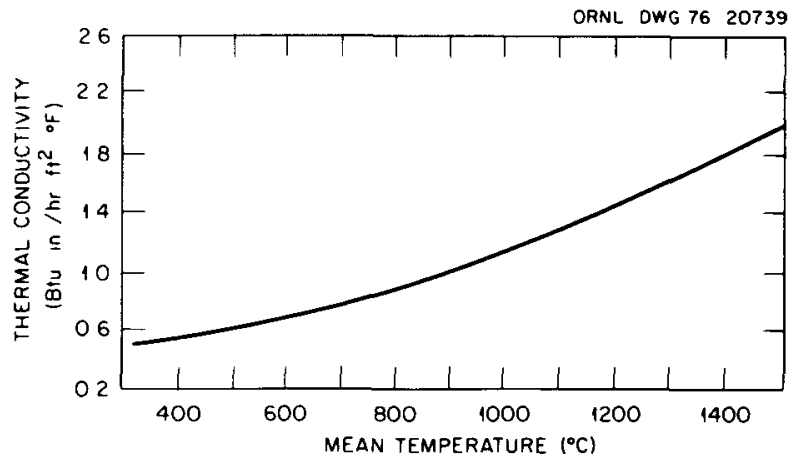

Fig. 10. Thermal conductivity of Kaowool 3000 in air as a function of mean temperature. $24 \mathrm{hr}$

$a_{\text {Time at }}$ the specified temperature is probably 
Table 10. Typical properties of Zircar board and Zircar zurconia fibers

\begin{tabular}{ll}
\hline \multicolumn{1}{c}{ Physicdl property } & \multicolumn{1}{c}{ Chardcteristics } \\
\hline Bulk density, $1 \mathrm{~b} / \mathrm{ft}^{2}$ & 30 \\
Porosity, $\%$ & 92 \\
Binders & None \\
Shrinkage, \% dfter $1 \mathrm{hr}$ at $3000^{\circ} \mathrm{F}$ & $0-10$ \\
Thermal expansion coefficient, & $6 \times 10^{6}$ \\
in $/ \mathrm{in} \cdot{ }^{\circ} \mathrm{F}$ & \\
Melting point, ${ }^{\circ} \mathrm{F}$ & 4700 \\
Maximum visable temperature, ${ }^{\circ} \mathrm{F}$ & 4000 \\
Compressive strength, psi & 42 \\
Fiber diameter, $\mathrm{mm}^{3}$ & $4-6$ \\
Fiber density, $\mathrm{g} / \mathrm{cm}^{3}$ & $5.6-59(92-97 \%$ dense) \\
Stabilizer & $\mathrm{Yttria}$ \\
Crystal phase & $\mathrm{Cubic}$ \\
Composition $\left(\mathrm{ZrO}_{2}+\mathrm{HfO}_{2}+\mathrm{Y}_{2} \mathrm{O}_{3}\right), \%$ & 99 \\
Thermal conductivity (in air), & \\
Btu $\times$ in $/ \mathrm{ft}^{2} \times \mathrm{hr} \times{ }^{\circ} \mathrm{F}$ & \\
$1000^{\circ} \mathrm{F}$ (mean) & 06 \\
$1500^{\circ} \mathrm{F}$ & 0.8 \\
$2000^{\circ} \mathrm{F}$ & 10 \\
$2500^{\circ} \mathrm{F}$ & 13 \\
$3000^{\circ} \mathrm{F}$ & 17 \\
\hline
\end{tabular}

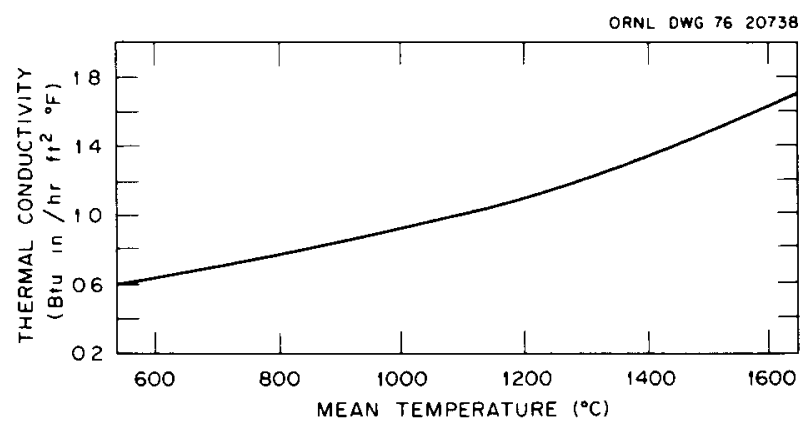

Fig. 11. Thermal conductivity of Zircar ZYFB3 in air as a function of mean temperature.

\subsection{Saffil Insulation}

Saffil is a trademark of ICI (Imperial Chemical Industries) Company for inorganic oxide fibers. Saffil alumina and Saffil zurconıa fibers are relatıvely pure alumina and zirconia. According to the manufacturer, there is no unfiberized shot present in Saffil fiber insulations. The average fiber diameter is $3 \mu \mathrm{m}$, the standard density is $6 \mathrm{lb} / \mathrm{ft}^{3}\left(0.096 \mathrm{~g} / \mathrm{cm}^{3}\right)$ Physical properties of Saffil alumina and Saffil zirconia fibers reported by the manufacturer are shown in Table 11. There are two grades of Saffil fibers currently on the market a standard grade and a high-temperature grade, which differ in density, surface area, and the recommended maximum usable temperature. The thermal conductivity data reported by the manufacturer are shown in Fig. 12.

The structure of Saffil fibers is polycrystallıne. ${ }^{21}$ Saffil alumına fibers as produced are $\gamma-\mathrm{Al}_{2} \mathrm{O}_{3}$ with a small percentage of other inorganic oxıdes. Saffil zirconıa fibers are $\mathrm{ZrO}_{2}$ stabilized in a tetragonal structure 
Table 11. Typical properties of Saffil fibers

\begin{tabular}{|c|c|c|}
\hline \multirow{2}{*}{ Physical property } & \multicolumn{2}{|c|}{ Characteristics } \\
\hline & $\mathrm{HT}$ & Standard \\
\hline \multicolumn{3}{|c|}{ Saffil alumina fibers } \\
\hline $\begin{array}{l}\text { F1ber density, } \mathrm{g} / \mathrm{cm}^{3} \\
\text { Melting point, }{ }^{\circ} \mathrm{F}\end{array}$ & $\begin{aligned} & 3.5 \\
> & 3600\end{aligned}$ & $\begin{aligned} & 3.0 \\
> & 3600\end{aligned}$ \\
\hline $\begin{array}{l}\text { Maximum-Use } \\
\text { temperature, }{ }^{\circ} \mathrm{F} \\
\text { Specific heat, Btu } / \mathrm{lb} \cdot{ }^{\circ} \mathrm{F} \\
\text { Tensle strength, psi } \\
\text { Young's modulus, ps } 1 \\
\text { Mean diameter, } \mu \mathrm{m} \\
\text { Surface area, } \mathrm{m}^{2} / \mathrm{g}\end{array}$ & $\begin{array}{l}2750 \\
0.25 \\
150 \times 10^{3} \\
15 \times 10^{6} \\
3 \\
1.5\end{array}$ & $\begin{array}{l}1850 \\
0.25 \\
150 \times 10^{3} \\
15 \times 10^{6} \\
3 \\
160\end{array}$ \\
\hline \multicolumn{3}{|c|}{ Saffil zirconia fibers } \\
\hline $\begin{array}{l}\text { Fiber density, } \mathrm{g} / \mathrm{cm}^{3} \\
\text { Melting point, }{ }^{0} \mathrm{~F}\end{array}$ & $\begin{array}{l}5.5 \\
>4900\end{array}$ & $\begin{array}{c}5.0 \\
>4900\end{array}$ \\
\hline $\begin{array}{l}\text { Maximum-use } \\
\text { temperature, }{ }^{\circ} \mathrm{F}\end{array}$ & 2900 & 2000 \\
\hline Specific heat, Btu $/ \mathrm{lb} \cdot{ }^{\circ} \mathrm{F}$ & 0.14 & 0.14 \\
\hline Tensle strength, psi & $100 \times 10^{3}$ & $100 \times 10^{3}$ \\
\hline Young's modulus, ps1 & $15 \times 10^{6}$ & $15 \times 10^{6}$ \\
\hline Mean diameter, $\mu \mathrm{m}$ & 3 & 3 \\
\hline Surface area, $\mathrm{m}^{2} / \mathrm{g}$ & 2.5 & 10 \\
\hline
\end{tabular}

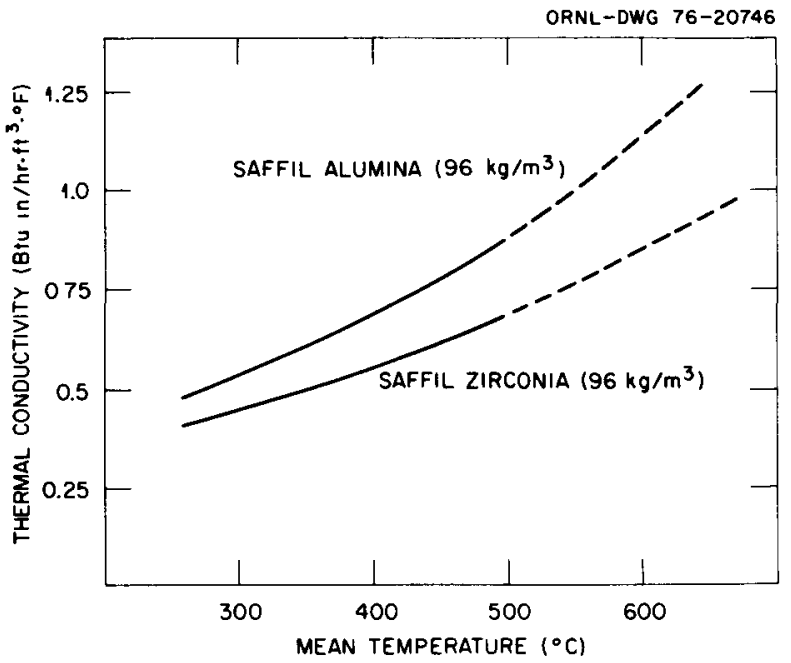

Fig. 12. Thermal conductivity of Saffil alumina and Saffil zirconia in air as a function of mean temperature. 
by the addition of a small percentage of other inorganic oxides. X-ray diffraction studies ${ }^{21}$ on Saffil alumina fibers indicate that the as-received material is $\gamma-\mathrm{Al}_{2} \mathrm{O}_{3}$ with five weak diffraction lines. After annealing at $1000^{\circ} \mathrm{C}$ for $500 \mathrm{hr}$, the major phase is still $\gamma-\mathrm{Al}_{2} \mathrm{O}_{3}$. At temperatures near $1200^{\circ} \mathrm{C}$, strong $\alpha-\mathrm{Al}_{2} \mathrm{O}_{3}$ peaks are observed. Saffil zirconia fibers as produced consist predominantly of a stabilized tetragonal phase plus a small amount of a cubic phase. At temperatures above $1400^{\circ} \mathrm{C}$, the monoclinic phase is present due to a phase transformation of cubic to monoclinic $\mathrm{ZrO}_{2}$.

Weight and dimensional changes as a function of temperature for fiber batts and blankets made from Saffil alumina and zirconia fibers were investigated by Strauss. ${ }^{21}$ His data are shown in Fig. 13(a) and $(b)$. At $1000^{\circ} \mathrm{C}$, Saffil fiber blankets exhibit good dimensional stability for times less than $500 \mathrm{hr}(<15 \%$ linear shrinkage was used as the stability criterion). Grain growth and sintering were observed at $1200^{\circ} \mathrm{C}$ for Saffil alumina and zirconia fibers. No significant changes in the fiber microstructure were observed after exposure at $1000^{\circ} \mathrm{C}$ for 100 to $500 \mathrm{hr}$. Saffil alumina fiber blanket annealed in air at 1000 to $1200^{\circ} \mathrm{C}$ for times up to $100 \mathrm{hr}$ has a lower thermal conductivity as compared with its initial value, probably due to the volume

(a)
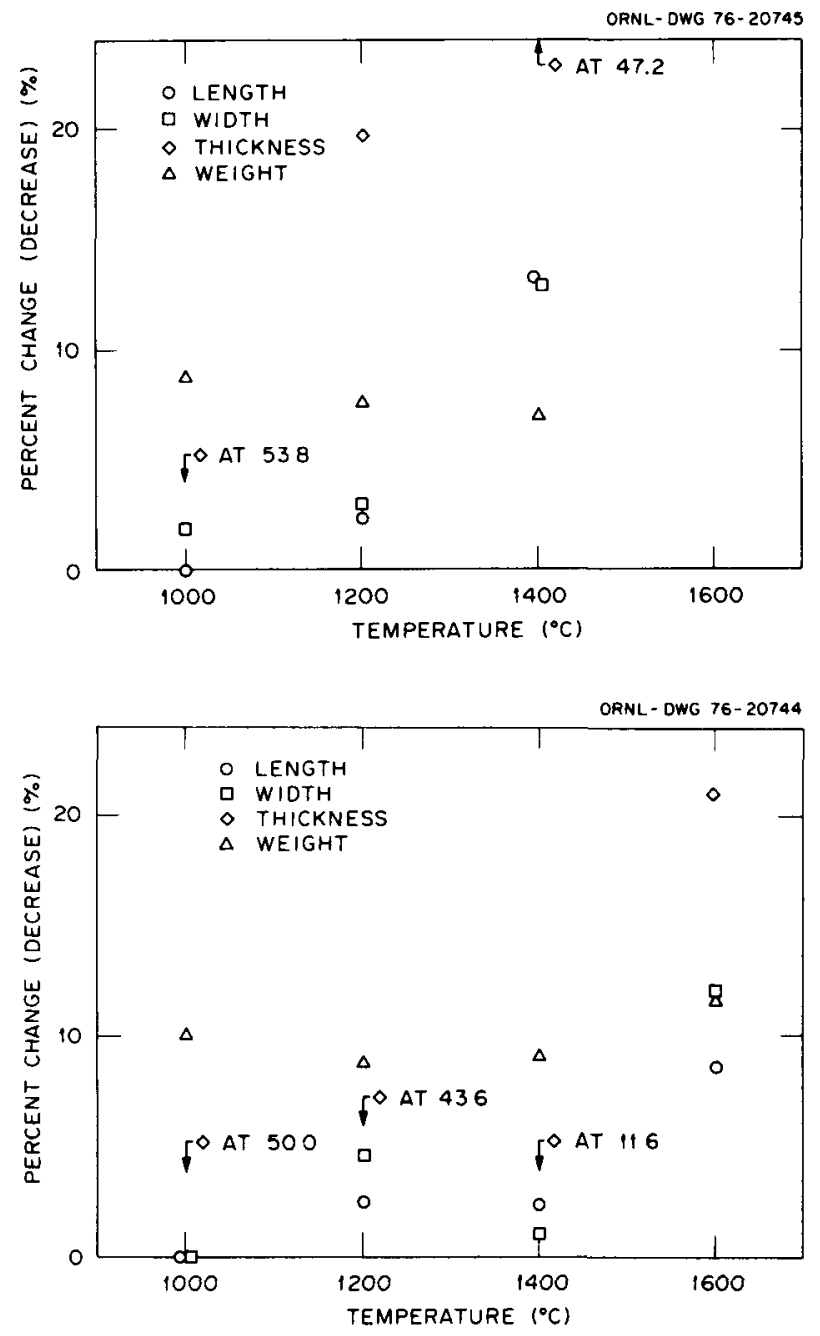

Fig. 13. Weight and dimensional changes in (a) Saffil zirconia HT blanket and (b) Saffil alumina HT blanket after 100-hr exposure to various temperatures. 
expansion of the fiber blanket But annealing at $1400^{\circ} \mathrm{C}$ for $500 \mathrm{hr}$ yields a thermal conductivity $65 \%$ higher than the initial value, primarily due to the shrinkage It was reported ${ }^{21}$ that Saffil alumina has the best dimensional stability among a selection of fibrous insulation blankets including Saffil alumina, Saffil zirconia, Fiberchrome, ${ }^{7}$ Kaowool 1400, Fiberfrax, B\&W Mullite, ${ }^{9}$ and Irish Refrasil

\subsection{Carbon Insulation}

Foam and fibrous insulations made of amorphous carbon are avalable in developmental and semicommercial quantities Physical properties of these materials are generally poorly known at present The manufactuer-reported thermal conductivity and the thermal conductivity measured by Dynatech R/D $\mathrm{Co}$ of one particular carbon foam material identıfied as $\mathrm{RVC}^{22}$ (reticulated vitreous carbon) are shown in Fig 14 The manufacturer's data make RVC material look promising for use in a hot segment of the insulation system if vacuum conditions would exist But Dynatech's thermal conductivity measurements showed a rather high thermal conductivity, which may exclude serious consideration of using this material in the selenide generator Table 12 lists some of the physical properties of the RVC material

22 Product of Chemotronics International, Ann Arbor, Mich

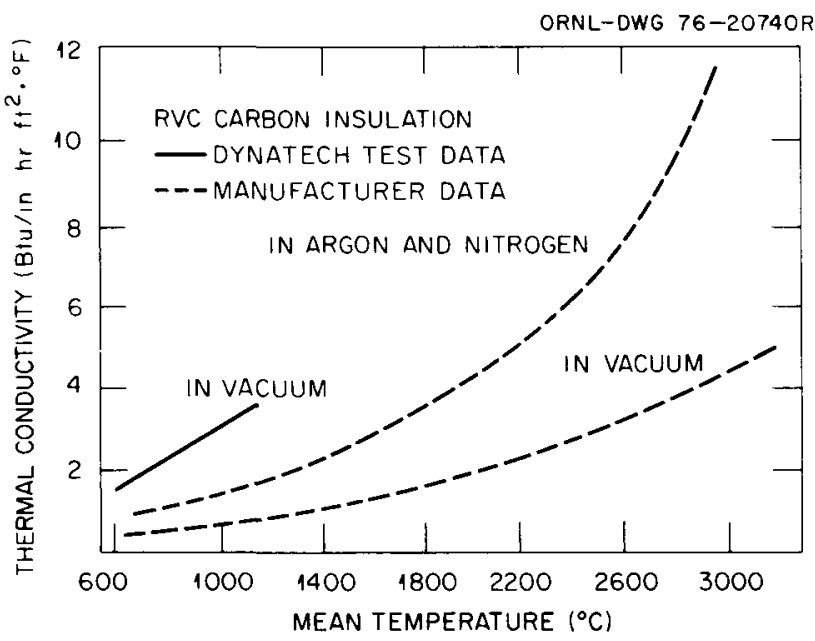

Fig. 14. Thermal conductivity of RVC insulation as a function of mean temperature.

Table 12. Typical properties of RVC-4 carbon insulation

\begin{tabular}{lc}
\hline \multicolumn{1}{c}{ Physical property } & Characteristics \\
\hline Bulk vold volume, $\%$ & 97 \\
Bulk density, $1 \mathrm{~b} / \mathrm{ft}^{3}$ & 31 \\
Crushing strength at & \\
$70^{\circ} \mathrm{F}$, psi & Varies with pore size 10 to 50 \\
Shrinkage to $2000^{\circ} \mathrm{F}$ & $\mathrm{N} 11$ \\
Volatiles to $2000^{\circ} \mathrm{F}$, & $\mathrm{N} 11$ \\
Melting point (sublimes), ${ }^{\circ} \mathrm{F}$ & 6330 \\
Temperdture limitation, ${ }^{\circ} \mathrm{F}$ & \\
In dir & 600 \\
In nonoxidizing atmosphere & 6330 \\
\hline
\end{tabular}


An oriented fibrous carbon insulation was developed by Ardary et al and measured by Godfrey et al. ${ }^{23}$ The material is a rigid, single-component, amorphous carbon material. Compressive strength is a function of density and is in the range of 100 to 500 psi. Thermal conductivity data avallable are shown in Fig. 15, it can be seen that the thermal conductivity of this material depends on the gaseous atmosphere, the heat treatment, and the fiber orientation. The thermal conductivity of this material is higher than the reported thermal conductivities of Kaowool 3000 and Zurcar type ZYFB3

\section{POTENTIAL APPLICATION PROBLEM AREAS}

In this section, potential problem areas associated with the use of fibrous insulations in the selenide generator are identified and discussed. A potential problem is associated with chemical reactions between the fibrous insulation and graphite. A large fraction of the hot-side fibrous insulation used in the converter and the heat source is in contact with graphite. Several of the candidate fibrous insulations for the selenide generator are based on silica or alumina-slica compositions. The reduction of slica or slicates by carbon is therefore of considerable importance Alumina or zirconia is also unstable with respect to carbon reduction

23 T G Godfrey, D L McElroy, and Z L. Ardary, Nucl Technol 22, 94 (1974)

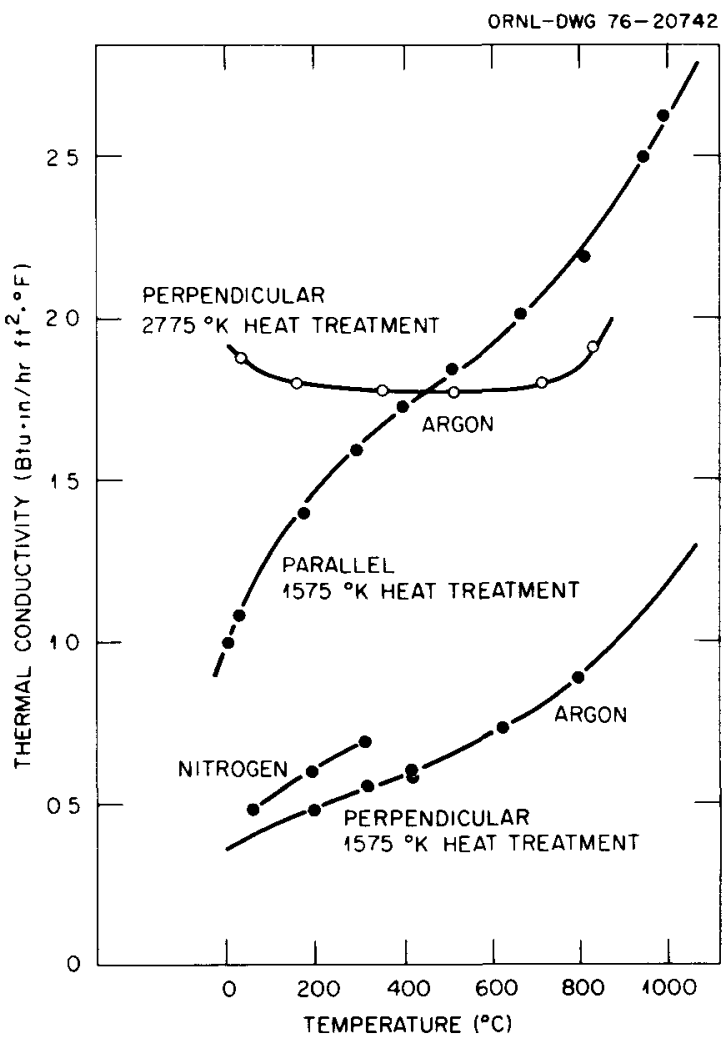

Fig. 15. Thermal conductivity of fibrous insulation of two different onentations and two heat-treatment temperatures. 
under the temperature and atmosphere conditions anticipated in the selenide sotope generator Silica can react with carbon as follows

$$
\begin{aligned}
\mathrm{C}+\mathrm{SiO}_{2} & \rightarrow \mathrm{S} 1+\mathrm{CO}_{2}(\mathrm{~g}), \\
\mathrm{C}+\mathrm{SiO}_{2} & \rightarrow \mathrm{S} \mathrm{O}(\mathrm{g})+\mathrm{CO}(\mathrm{g}), \\
\mathrm{CO}(\mathrm{g})+\mathrm{S}_{2} \mathrm{O}_{2} & \rightarrow \mathrm{S} 1 \mathrm{O}(\mathrm{g})+\mathrm{CO}_{2}(\mathrm{~g})
\end{aligned}
$$

The standard free-energy changes for reactions (1), (2), and (3) can be calculated from the standard enthalpy and entropy of the reactant and the product phases shown in Table $13^{24}$

$$
\begin{aligned}
& \Delta G_{1}^{\circ}=122,950-442 T \mathrm{cal} / \mathrm{mole}, \\
& \Delta G_{2}^{\circ}=167,400-865 T \mathrm{cal} / \mathrm{mole}, \\
& \Delta G_{3}^{\circ}=126,150-444 T \mathrm{cal} / \mathrm{mole}
\end{aligned}
$$

The standard free energy changes for reactions (1), (2), and (3) can also be expressed in terms of the equilibrium partial pressures of various gaseous species, $P_{\mathrm{S}_{1} \mathrm{O}}^{\circ}, P_{\mathrm{CO}}^{\circ}, P_{\mathrm{CO}_{2}}^{\circ}$

$$
\begin{aligned}
& \Delta G_{1}^{\circ}=-R T \ln P_{\mathrm{CO}_{2}}^{\circ}, \\
& \Delta G_{2}=-R T \ln P_{\mathrm{S}_{1} \mathrm{O}}^{\circ} P_{\mathrm{CO}}^{\circ}, \\
& \Delta G_{3}=-R T \ln P_{\mathrm{S}_{1} \mathrm{O}}^{\circ} P_{\mathrm{CO}_{2}}^{\circ} / P_{\mathrm{CO}}^{\circ}
\end{aligned}
$$

Equating these two sets of equations at $930^{\circ} \mathrm{C}$ yields

$$
\begin{aligned}
P_{\mathrm{CO}}^{\circ} & =908 \times 10^{-5} \text { torr }, \\
P_{\mathrm{CO}_{2}}^{\circ} & =160 \times 10^{-10} \text { torr }, \\
P_{\mathrm{S}_{1 O}}^{\circ} & =26 \times 10^{-5} \text { torr }
\end{aligned}
$$

If the design atmosphere conditions in the converter and the heat source have partial pressures of $\mathrm{CO}, \mathrm{CO}_{2}$, and $\mathrm{S} 1 \mathrm{O}$ lower than the above equilibrium partial pressures, reactions (1) and (2) will proceed While the effect of reactions (1) and (2) on fibrous insulation might be small, the silicon-bearing species $\mathrm{S} 1 \mathrm{O}(\mathrm{g})$ can potentially deposit on cooler generator components and could embrittle the IHF (isolation hot frame)

24 JANAF Thermochemical Tables, 2d ed, NBS-37, National Bureau of Standards, Washıngton, D C , 1971

Table 13. Thermochemical parameters of several species of interest

\begin{tabular}{lccccccc}
\hline Species/parameter & $\mathrm{C}$ & $\mathrm{Si}$ & $\mathrm{SIO}_{2}$ & $\mathrm{CO}_{2}$ & $\mathrm{CO}$ & $\mathrm{S} \mathrm{O}$ & $\mathrm{SIC}$ \\
$\Delta H_{298}^{\circ}, \mathrm{kcal} / \mathrm{mole}$ & 0 & 0 & -2170 & -9405 & -2640 & -2320 & -15 \\
$\Delta S_{298}^{\circ} \mathrm{kr} / 1 / \mathrm{mole}^{\circ} \mathrm{K}$ & $1361 \times 10^{3}$ & $45 \times 10^{3}$ & $10 \times 10^{3}$ & $511 \times 10^{3}$ & $473 \times 10^{3}$ & $5055 \times 10^{3}$ & $395 \times 10^{3}$ \\
\hline
\end{tabular}


material Pt-3008 (Pt-Rh-W alloy, 62\% Pt, 30\% Rh, 8\% W). ${ }^{25}$ It has been reported that $10 \mathrm{ppm}$ of silicon can generate a second phase at grain boundarles of Pt-3008, and $30 \mathrm{ppm}$ of silicon in the bulk can embrittle Pt-3008 ${ }^{26}$ It seems that, in terms of compatibility with graphite and Pt-3008, pure alumina or zirconia fibers are better material selections than silica-based fibers for use as thermal insulations in the selenide isotope generator. The above calculations assume a constant temperature, $930^{\circ} \mathrm{C}$. If the operating temperature is higher, this compatıbility problem will be severe because of higher equilibrium partial pressures of $\mathrm{CO}, \mathrm{CO}_{2}$, and $\mathrm{SiO}$

In the thermoelectric modules, fibrous insulations are in intimate contact with the selenide legs. Selenide thermoelectric elements have appreciable vapor pressures at the operating conditions The equilibrium partial pressures ${ }^{27}$ of selenium-bearing species over TPM-217 P-type material ${ }^{28}$ are shown in Fig. 16. One potential material problem in the system is the reaction of selenium with fibrous insulations, with the consequence of degradation and structural instability in both thermoelectric elements and fibrous insulations. There are currently no published data on this subject.

Insulation outgassing is always an important step in preparing fibrous insulations prior to installation in critical generator applications. Due to the high specific surface area (area/unit mass) inherent to all fibrous

25 C T Liu and H Inouye, Novel Platınum-Rhodium-Tungsten Alloy, U S Patent 3,737,305, June 5, 1973

26 A C. Schaffhauser, Isotoptc Power Matenals Development Quarterly Progress Report for Pertod Ending June 30 , 1976, ORNL/TM-5569

27 E F. Hample, R B Ericson, W C Mitchell, and R S Reylek, "TPM-217 Materidl Performance Characteristics," paper No 729040 presented at the Intersociety Energy Conversion Engineering Conference, San Diego, Calıf, September 1972

28 E F Hample, P-Type Thermoelectric Element, U S Patent 3,853,632, Dec 10, 1974

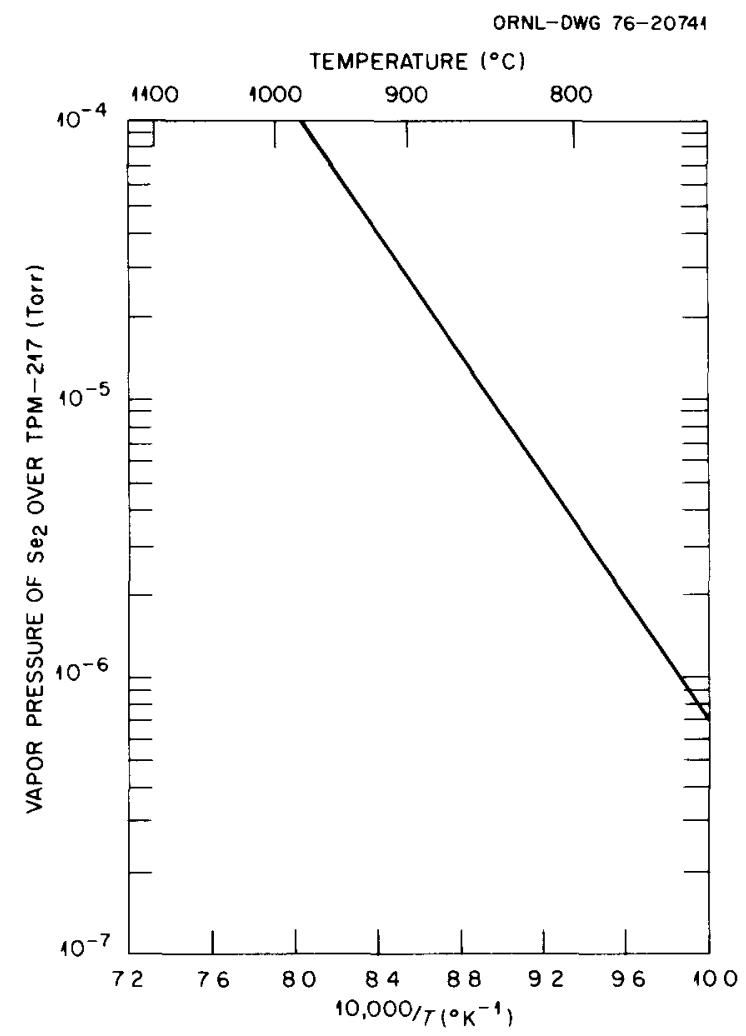

Fig. 16. Vapor pressure of $\mathrm{Se}_{2}$ over P-type TPM-217. 
materials, the amount of moisture and other absorbed gases in even a relatively small mass of insulation is substantial. Water vapor absorbed on fibers can increase the thermal conductivity significantly. This is because water vapor desorbs at high temperature, migrates to and possibly condenses at relatively lower temperatures, and thereby incurs a heat transport. Other gases $\left(\mathrm{CO}, \mathrm{CO}_{2}, \mathrm{~N}_{2}\right)$ adsorbed on fibers can also desorb at high temperatures and thus increase the heat conduction plus convection through a gas phase. Because of these possibilities it is always considered necessary to carefully outgas fibrous insulations before use. Optimum outgassing procedures for various fibrous insulations have not been fully established and are usually determined empirically.

After outgassing, fibrous insulations must be stored and processed in an inert dry atmosphere such as purified argon to minimize readsorption of various species in the insulation. Great care should be placed on cutting, punching, or drilling fibrous materials. There is at least one report ${ }^{29}$ on the contamination of thermoelectric elements due to impurities in fibrous insulations picked up during the process of drilling holes.

Small concentrations of other oxides are commonly added to relatively pure fibers to stabilize the fibrous structure. While the impurities are important constituents in the fibers for the purpose of structural stabilization, they may result in compatibility problems to other components in the selenide generator. Impurities such as $\mathrm{Na}, \mathrm{K}, \mathrm{Fe}, \mathrm{Cr}, \mathrm{B}, \mathrm{Cl}$, and $\mathrm{S}$ have relatively high diffusivities in oxide fibers at high temperatures. They tend to diffuse out of the fibers at the hot side of fibrous insulations. Some of these impurities have been proven detrimental to at least the IHF alloy Pt-3008, ${ }^{26}$ and possibly to the selenide elements.

Thermal insulation stability of fibrous insulations depends sensitively on the structural and microstructural stability. For long times at high temperature, fibers based on silica can devitrify or crystallize. For instance, amorphous silica fibers can transform to cristobalite, which then transforms to quartz at temperatures lower than $1093^{\circ} \mathrm{C}$. These transformations are accompanied by significant dilation and mechanical cracking. A schematic stability diagram of stable and metastable forms of silica is shown in Fig. 17. Numerous studies on the devitrification of fused silica have been reported on bulk specimens. In

29. J. O. Collins, Develop 1800F-400F Fibrous-Type Insulation for Radioisotope Power Systems, phase 1 final report, vol. 1, ALO-3633-7 (1967).

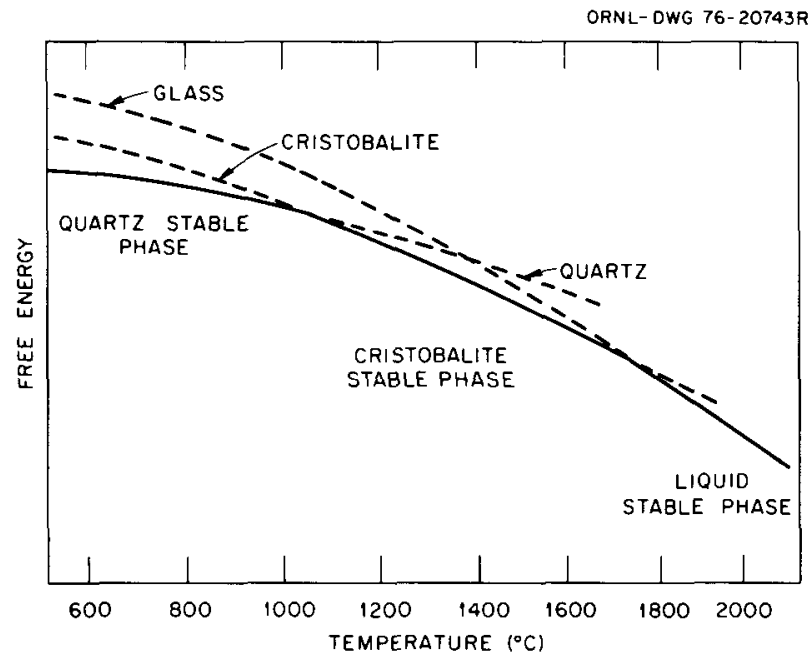

Fig. 17. Relative free energy of various forms of silica. 
the case of fibrous materials, the degree of devitrification is expected to be more extensive than in bulk specimens due to the fact that the avalable high surface area of the fibers provides numerous nucleation sites for devitrification Devitrification in Microquartz fibers has been studied, ${ }^{30}$ and experimental data are shown in Fig 18 Devitrification kinetics are highly dependent upon the presence of impurities, especially water, and the surface conditions of the fibers

Phase transformations can occur in alumina and zirconia fibers when they are maintained at high temperatures for a sufficiently long time Grain growth of each crystalline grain and sintering between fibers can be detrimental to the fibrous structure, and volume changes associated with phase transformations will generate cracks and thus cause fiber fragmentation This process can result in additıonal shrinkage Sintering at high temperatures will also cause shrinkage While shrinkage in fibrous insulations might decrease the thermal conductivlty due to more gas phase convection barners plus more radiation barriers per unit volume, the total thermal conductance of the insulation system is usually increased due to the shrinkage in the thermal insulation thickness In addition, crystalline phases resulting from devitrification will increase the thermal conductivity of the solid phase because the phonon mean free path is longer in crystalline lattices than in amorphous phases, and the conductivity of the solid phase is roughly proportional to phonon mean free path All these factors should be considered in the evaluation of a suitable fibrous insulation for a critical application such as the selenide generator There is presently a lack of both qualitative and quantitative information in areas of devitrification, grain growth, and sintering of the candidate fibrous insulations discussed previously

$30 \mathrm{~J}$ I Mueller et al, Fundamental Studies on the Nature and Properties of Ceramic Fiber Insulation NASA CR 137793 (July 1975)

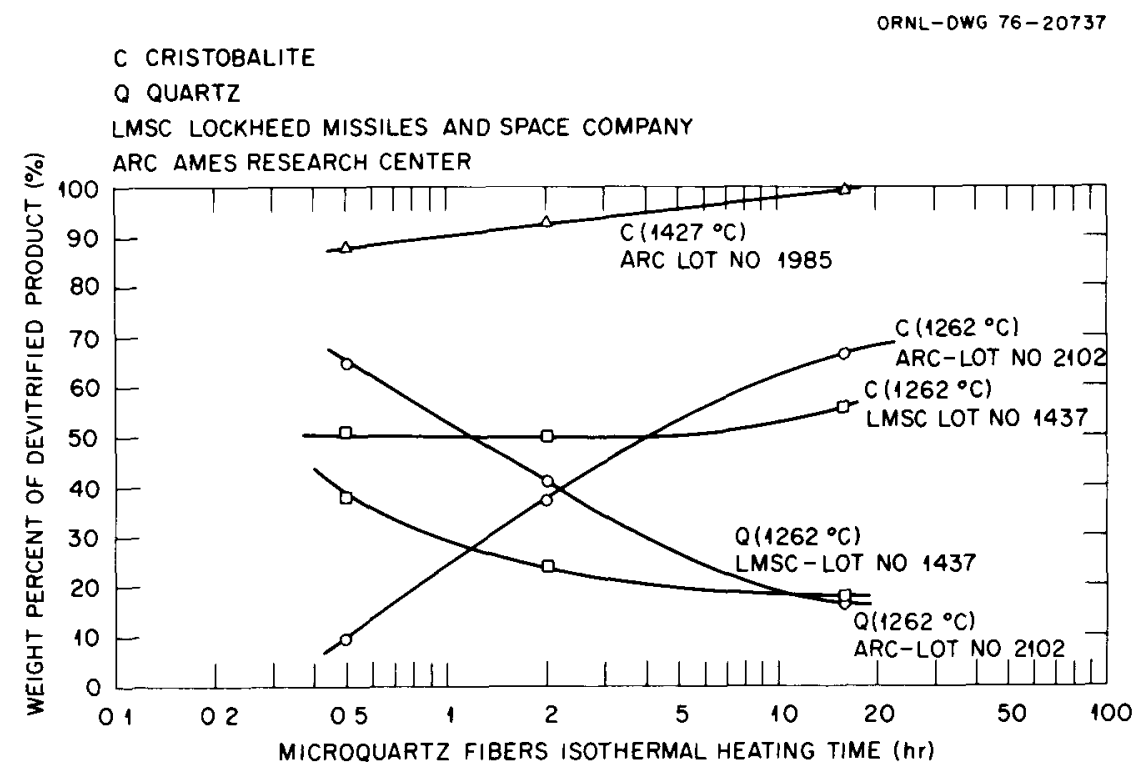

Fig. 18. Weight percent of devitnfied product in Microquartz fibers as a function of isothermal heating time, temperature, and different sources of raw matenals. 


\section{CONCLUSIONS}

The conclusions of this report are summarized as follows

1 Thermal conductance of fibrous insulations which have the potential to be used in the selenide generator must be measured in a manner simulating the application conditions. The thermal conductivity data supplied by the manufacturers should be used only as a guideline

2 Compatibility between fibrous insulations and graphite, $\mathrm{Pt}-3008,{ }^{26}$ plus the thermoelectric elements should be confirmed before the GDS tests.

3. Information on the fiber's structural and microstıuctural changes, at high temperatures for times approximating the service hife, including devitrification, sintering, and grain growth, is presently lacking. Further work in these areas is needed to justify long-term use of fibrous materials

4. Outgassing, storage, forming, and installation of fibrous insulations are all essential to the effectiveness, uniformity, and compatibility of the insulation system. The prepardtion procedures at present are not fully established.

5. The high temperatures in the nonmodule areas of the thermoelectric converter and at the ends of the heat source require an insulation capable of sustaining a moderate compressive load. Potential candidates at present include Cotronics 360, Kaowool 3000, Zircar ZYFB3, and carbon fibers. Determination of phases present, especially the binder phase, measurement of the effective thermal conductance, and experiments on the structural and chemical stability of these fibrous materials are required to provide a sound technological basis for selecting the insulation material for this load-bearing application.

o Characterization of commeicid fibious matenals by chemical analysis, scanning elertinn microscopy, and $\mathrm{x}$-ray analysis is imperative, because the impurity content, concentiation of organic or morganic binders, shot content, fiber dimension and distribution, and fiber structure are all basic factors contributing to the low thermal conductivity, good structural and dimensional stablity, and hence insulating stability of the fibrous material

7 The resilience or spring-back properties of fibrous insulations should be tested to verify the dimensional and structural stability under the design compression at design temperature 
INTERNAL DISTRIBUTION
1 R G Donnelly
2 G M Goodwin
3 D E Harasyn
4 R L Heestand
5-7 M R Hill
8 H Inouye
9 J R Keiser
10 C T Liu
11 O L McElroy
12 H Postma
13-17 A C Schaffhauser

\author{
18 G M Slaughter \\ 19-23 V J Tennery \\ 24 D B Trauger \\ 25-29 G C We1 \\ 30 J R Weir \\ 31 C L White \\ 32-33 Central Research Library \\ 34 Document Reference Section \\ 35-39 Laboratory Records \\ 40 Laboratory Records, ORNL RC \\ 41 ORNL Patent Office
}

\section{EXTERNAL DISTRIBUTION}

42 AlResearch Manufacturing Company of Arizona, MS 93-240-503-3, 402 South 36th Street, P O Box 5217, Phoenix, AZ 85010

J E McCormick

43-53 ERDA Division of Nuclear Research and Applications, Washington, DC 20545
R C Brouns
J S Griffo
G A Newby
W D Kenney
B J Rock
A P Litman
C O Tarr
J J Lombardo
$\mathrm{N}$ R Thielke
E J Wahlquist

A L Mowery

54 ERDA Oak Ridge Operations Office, P O Box E, Oak Ridge, TN 37830

Research and Technical Support Division

55-56 General Electric Company, Nuclear Programs, P O Box 8661, Philadelphıa, PA 19101
E H Sayell
E W Willams

57-58 Los Alamos Scient1fic Laboratory, P O Box 1663, Los Alamos, NM 87545
S E Bronisz
S S Hecker

59 Minnesota Mınıng and Manufacturnng Company, 3M Center, Bldg 260 5B-6, St Paul, MN 55101

E F Hampl

60-61 Monsanto Research Corporation, P O Box 32, Miamısburg, OH 45342
D L Coffey
E W Johnson 
62. Sunstrand Energy Systems, 4747 Harrison Avenue, Rockford, IL 61101

E. Kreuger

63-65. Teledyne Energy Systems, 110 W. Timonium Road, Timonium, MD 21093

W. J. Barnett W. Osmeyer

A. R. Lieberman

66-92. Technical Information Center 